\title{
Atomic Genetics and Origin of the Universe- Volume-8
}

\author{
V. M. DAS* \\ University Of God, Das Nursing Home, Parade Ground, Fatehgarh, 209601, India
}

\begin{abstract}
It is being believed that only three times nucleosynthesis did take place after the origin of universe by Big Bang Firstly, after Big bang ( $\mathrm{H}$ and He ), secondly on star ( up to iron )and thirdly in supernovae explosion ( beyond $\mathrm{Fe}$ ). While studying solar system and earth formation, it has been found that all elements of planets, satellites, asteroids were formed by unconditioned working of nature i.e. they were directly formed by from proto planets, proto satellites and proto asteroids. Hence we are not star dust. Decay of particles should be studied in the light of structure of the particles and in the light of dark matter and dark energy which form during the decay of particles both annihilation and materialization. The equation on both the side should be balanced as regard total number of fermions and bosons involved in particle annihilation and materialization or interactions. The spin property of particles is very important property as all physical interactions, chemical interactions and life sciences interactions are maintained by transfer of different messages from one unit to another unit. Had there been no spin of electron, nucleus of atoms, the all interactions would be no more and the structure and functions of atoms, planets, solar system, galaxies, and visible and invisible universe would collapse. Einstein theory of relativity states that nothing could move more than velocity of light. There are two phenomena which are FTL ( faster than light) phenomena. Firstly is prayer phenomenon and the secondly is pre creation era when programming of universe was triggered by Almighty B.B.B. Had there been no programming of universe in pre creation era, there would have been no creation, no oscillation phenomenon of the universe. It is the mind that decides energy matter interactions. But it is the stimulus ( strength of stimulus) that decides ( conditioned stimulation of thought) the type of thought to be expressed in energy matter interaction. Compton effect, photo electric effect and pair production, these are three types of energy matter interactions which have different effects as they are triggered by different strength of stimulus leading to different thoughts expression to give that different effects . Abundance of helium in universe or in proto star formation does mean that He had formed after Hydrogen ( H2). But in quasars, the ejected clouds were free of helium and other heavy elements. It means that He had formed by unconditioned way of nature before formation of proto stars .
\end{abstract}

\section{Introduction}

It is the mind that triggered nucleosynthesis on , planets, satellites and asteroids separately by unconditioned working of nature. While on sun and supernova blast necleosynthesis takes place by conditioned working of nature. It is the mind that decides the type of decay of particles. It is the mind that decides the type of spin of particles. If the thought of integer spin is there the particle would exhibit integer spin ( all bosons ) or if the thought of half integer spin is there the particles ( all fermions ) would exhibit half integer spin. But energized gravitons though they are fermions have integer spin. Prayer is the phenomenon that is FTL phenomenon . It has been confirmed that prayer works .

\subsection{Nucleosynthesis [1]}

\section{Structure}

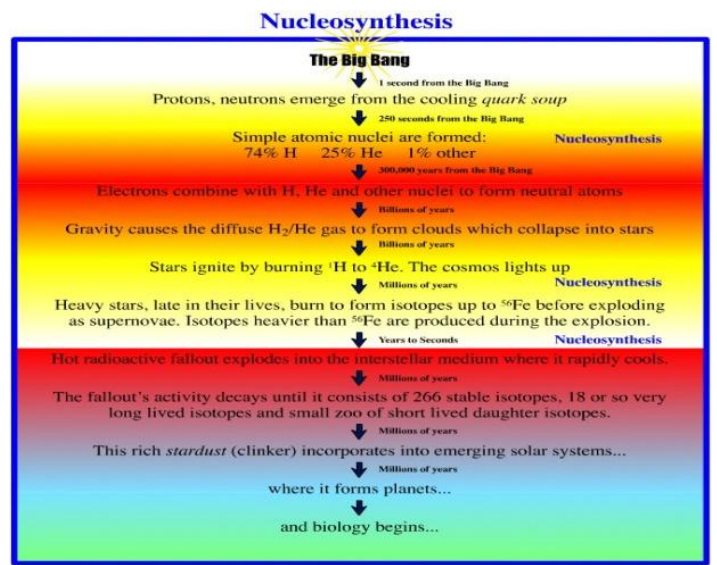

(Figure 1 wrong depiction of nucleosynthesis [1] ) 
To understand phenomenon of nucleosynthesis on quasars, stars, planets, satellites ,asteroids, we have to understand about how does nature work ( Figure 2 ), conditioned and unconditioned expressions of atomic genes ( Figure -3) .

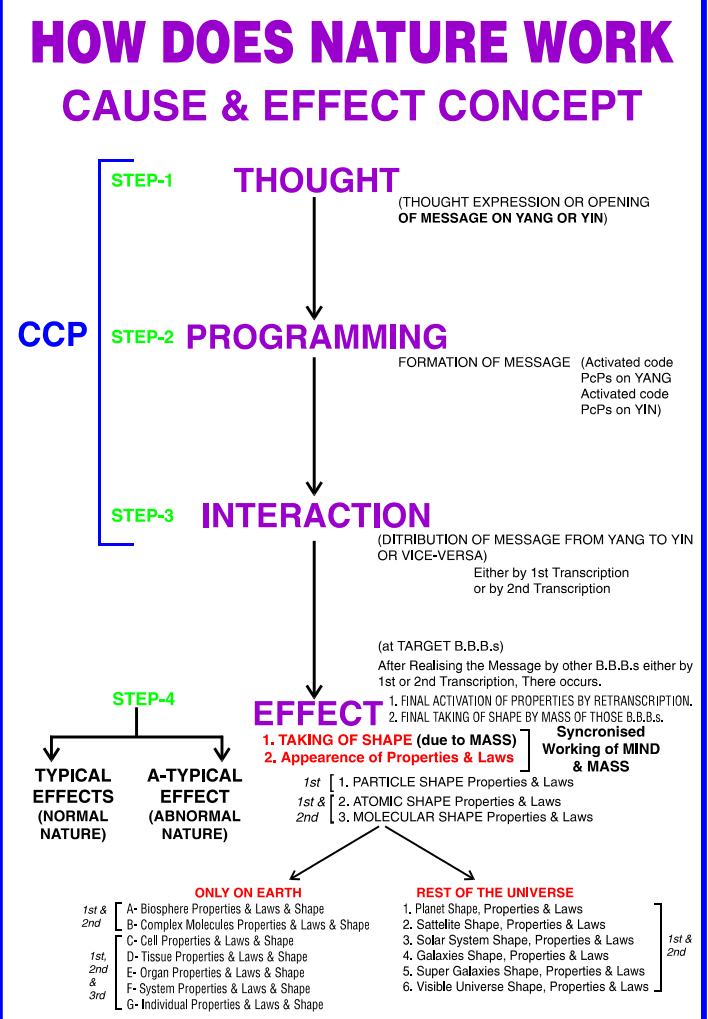

( Figure 2 How does nature work )

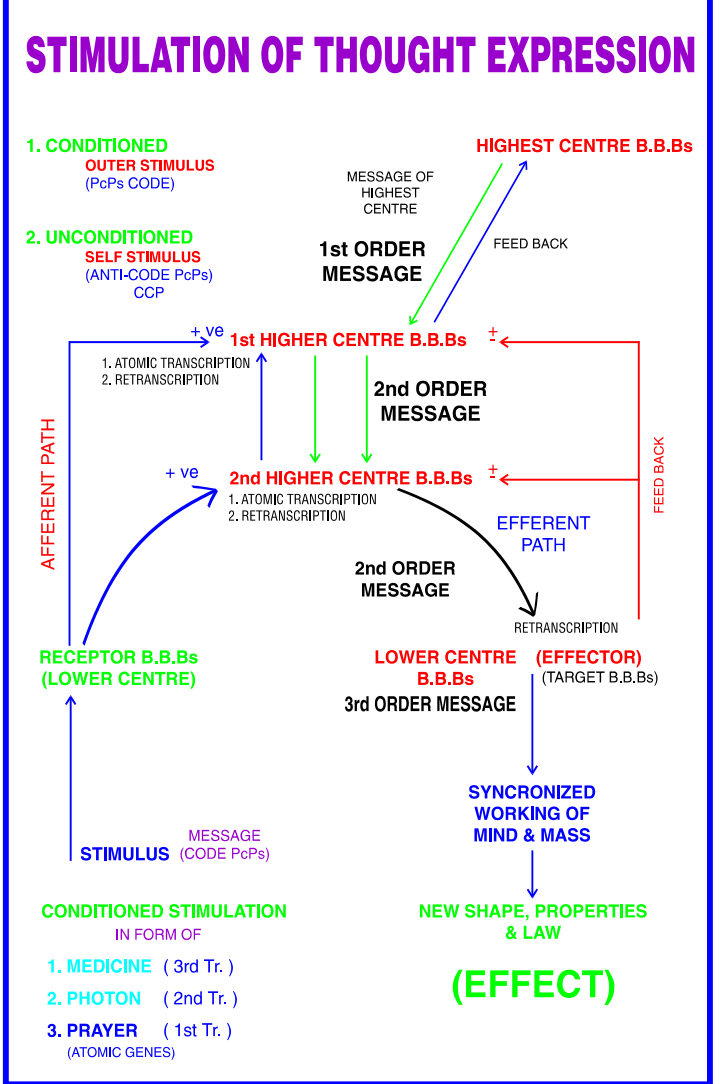

(Figure 3 stimulation of thought expressions ) 
Origin of the Earth ( figure 4 ) - Till today it is believed that earth is the part of sun and sun is second generation star..There are observations which contradict this hypothesis. After the formation of rotating GMC (giant molecular cloud), there developed many nidus (B.B.Bs working as higher centers) in the rotating nebula. These higher centers expressed thoughts of condensation of nebula in different sectors that will give rise to formation of proto stars, protoplanets, proto satellites and proto asteroids and thoughts of contraction or collapse that will cause shrinkage of proto star, protoplanets and proto satellite. The formation of asteroid belt is due to thought expression of clumping or collapse. Thus there developed many different sectors in same nebula. Along with this the spin thoughts were also triggered. This led to development of spin of proto star, protoplanets, proto satellites and proto asteroids. Finally the nebula transformed into proto star, protoplanets, proto satellites and proto asteroids. About 4.6 billion years ago, formation of our solar system took place.( Figure 4) In these proto star, protoplanets, proto satellites, proto asteroids many other different thought expressions were expressed by many other higher centers (B.B.Bs) that had led to formation of sun, different planets, different satellites and asteroid belt between mars and Jupiter. ALL ARE BORN FROM SEPARATE NEBULAR SECTORS AND THUS THEY ARE CREATED WHERE THEY ARE PRESENT NOW IN ORBIT. They all have their different shapes, size, different properties, different compositions and behavior which were triggered by many different higher centers.. The asteroid belt present between Mars and Jupiter was created from proto asteroid by phenomenon of clumping. THIS WAS ALL DUE TO UNCONDITIONED THOUGHT EXPRESSIONS OF B.B.Bs.(Figure 3)

\section{Origin of Solar system ( figure 4)}

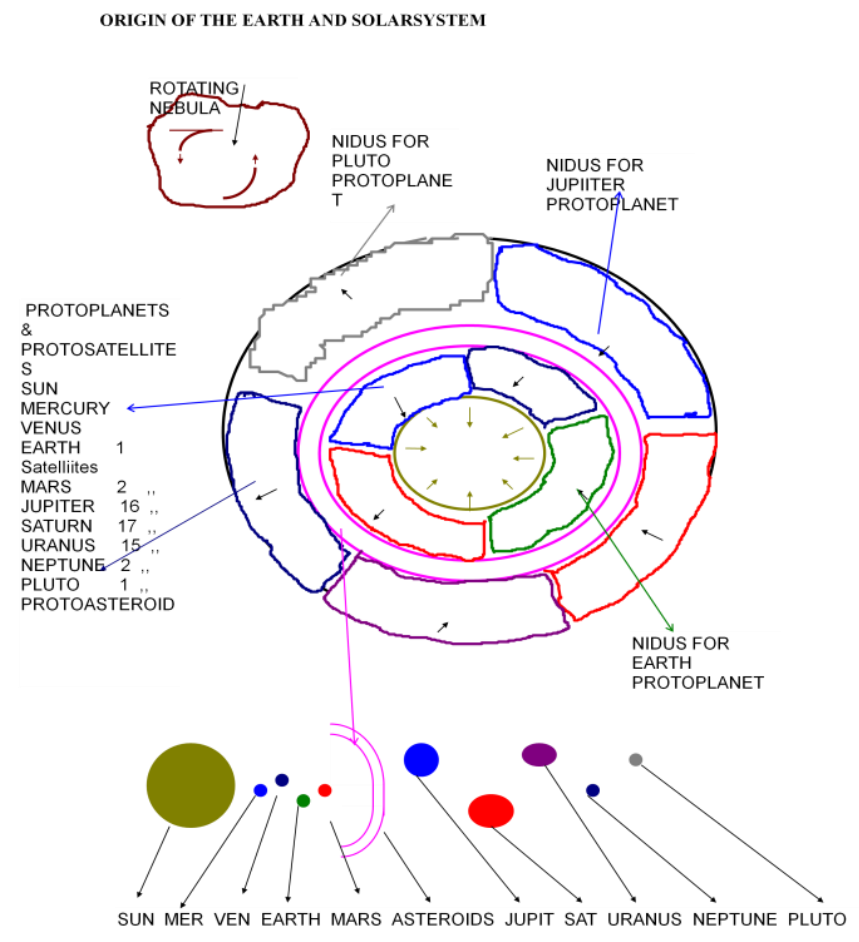

\section{( figure -4 origin of solar system)}

\section{Origin of continents and their shifting ( figure 5)}

When the Earth was forming, there developed many other higher centers for different thought expressions. These were--

1. Thought expressions of formation of continents and water.

2. Thought expressions of formation of different layers of the earth. ( figure 6 )

3. Thought expressions of formation of different layers of atmosphere.

And thus Pangaea continent, sea, different layers of earth, and different layers of atmosphere were formed by target B.B.Bs. (Figure 5 ) Simultaneously there developed other higher centers which expressed following thoughts-- 
1. Thought expression of ignition of the core

2. Thought expression of magma.

3. Thought expressions of shifting of continents.( Figure 5 )

4. Thought expressions of seismic foci

5. Thought expression of water cycle

And thus formation of heat in the core, magma in mantle layer, seismic foci in lithosphere, shifting of different continents in their respective directions and appearance of water cycle took place by different target B.B.Bs. This was all triggered by unconditioned working of B.B.Bs. The entire transmutation took place simultaneously.
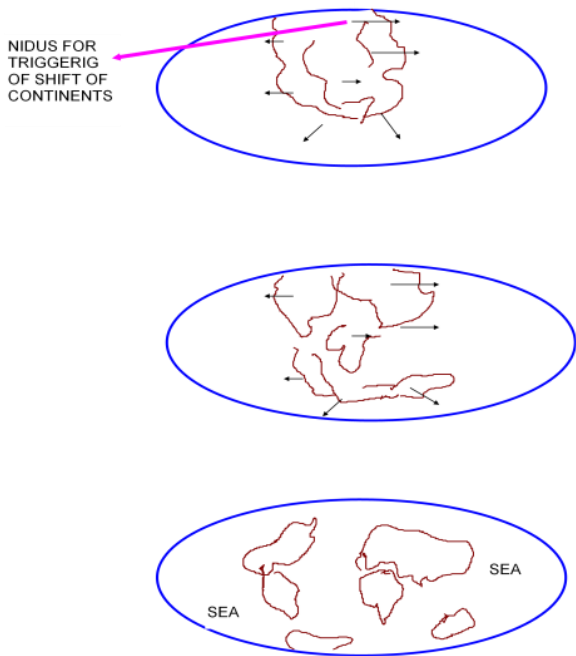

Figure 5 Origin of continents and their shift

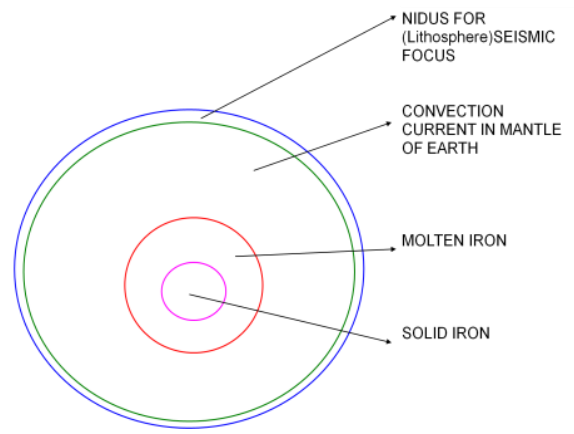

( Figure 6 Layers of Earth )

Having equipped with some knowledge of origin of universe, origin of solar system, origin of earth and moon , and asteroid belt ( Figure 4 ), it is now clear that all these celestial bodies were formed separately from the rotating nebula made up of hydrogen and helium. These all celestial bodies were formed directly from nebula by unconditioned working of nature . Hence nucleosythesis took place by unconditioned working of thought expressions while these celestial bodies were forming except sun.

In quasars where apart from formation of hydrogen atoms from tachyons, nature also formed some other elements from created hydrogen by unconditioned working of thought expressions. If we see spectrum of quasars, we could see those elements which are being created there.

Quasar redshifts are measured from the strong spectral lines that dominate their optical and ultraviolet spectra. These lines are brighter than the continuous spectrum, so they are called 'emission' lines. They have widths of several percent of the speed of light. These widths are due to Doppler shifts caused by the high speeds of the gas emitting the lines. Fast motions strongly indicate a large mass. Emission lines of hydrogen (mainly of the Lyman series and Balmer series), helium, carbon, magnesium, iron and oxygen are the brightest lines. The atoms emitting these lines range from neutral to highly ionized, i.e., many of the electrons are 
stripped off the ion, leaving it highly charged. This wide range of ionization shows that the gas is highly irradiated by the quasar, not merely hot, and not by stars, which cannot produce such a wide range of ionization. Iron quasars show strong emission lines resulting from low ionization iron (FeII), such as IRAS 18508-7815. [2]

This type of nucleosynthesis is being taking place while creation is going on. Hence we can assume that nucleosyntheis could be made by unconditioned working of thought expressions.

When this GMC that was created in the quasar ejected out in the closed universe, where hydrogen and helium were predominantly in high percentage, transformed into proto stars, proto planets and proto satellites and proto asteroid by unconditioned working of thought expressions.

In stars, with the triggering of nuclear fusion, there started again formation of new elements. Now this time it was conditioned working of thought expression which could lead to new nuclei formation .

But on planets and satellites, and in asteroid belt, nucleosynthesis took place by unconditioned thought expressions and with the result during the formation of planets and satellite and asteroid belt, new atoms were created, which formed them and they are still existing on that celestial bodies. These atoms were joined to form molecules, complex molecule of life and finally living cells by unconditioned working of thought expression. Life is nothing but higher thought expressions of B.B.Bs . Formation of simple molecules are due to lower thought expressions of theses B.B.Bs.

\section{Conclusions}

1. It is believed that first nucleosynthesis ( $\mathrm{H}$ and $\mathrm{He}$ ) took place after the Big bang. Second nucleosynthesis ( upto $\mathrm{Fe}$ ) took place during star formation when nuclear fusion started and last nucleosyntheis ( beyond $\mathrm{Fe}$ ) took place during supernova explosion. Only three times nucleosynthesis took place during formation of solar system and Earth. It is all Myth and illusion .

2. Quasars are believed to be the ANG( active nuclear galaxies ) and they are equipped with black holes . It is all Myth and illusion.

3. Earth is part of Sun and sun is second generation star and moon is the part of earth and it was formed by collision of earth with some passing celestial object. It is all myth and illusion.

4. Earth is the part of sun as it is hot inside and crust ( Land and water ) of earth formed by cooling of the surface . Continental shift is due to strike of big asteroid or comet that led to their shifting . It is all myth and illusion.

5. Earth quakes are due to Global plate tectonic movement and collisions. It can never be predicted and prevented. Hence it is a permanent defect in earth which could never be corrected. It is all Myth and illusions .

\section{Discussion}

1.Having equipped with knowledge of Mind and Mass It is now clear unless mind triggers, nature cannot give effect of nucleosynthesis . Triggering of Mind is of two types one is conditioned i.e. outer stimulus triggers nucleosynthesis that happens on Sun and supernova explosion. Second one is unconditioned triggering of Mind i.e. self stimulation of nucleosynthsis that happened in quasars, planets, moons and on asteroids. All thoughts are fed thoughts and this feeding was done by Highest center of the universe in pre creation era.

2. The Law of nucleosynthesis states that there is one law in nucleosynthesis that there is no law except wish of Almighty or highest center of universe that programmed our universe during pre creation era. Hence periodic table ( number of elements and their amount or percentage ) of Quasars, Suns, supernova explosion, planets, moons, asteroids would never be same.

3. We are NOT star dust.

\subsection{Phenomenon of decay of particles}

To understand laws of decay of subatomic particles, we should understand first laws of transmutation because decay is nothing but a process of transmutation. In biological world, we observe this phenomenon first to understand the process of decay.

1. Any transformation of animal protein into our body protein, it first breaks down in to its basic constituent or basic unit or more fundamental units which are known as amino acids. The number of amino acids absorbed in digestion before transformation are equal to number of amino acids after transformation of body protein. These transformations are taking place in presence of enzymes .

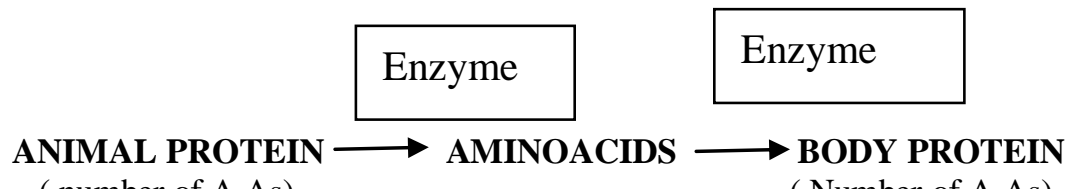


Hence any particle transforming into other particles must follow this rule i.e. to break into its fundamental units and thought is necessary to make this transformation complete. Unless thought is expressed, transformation cannot occur.

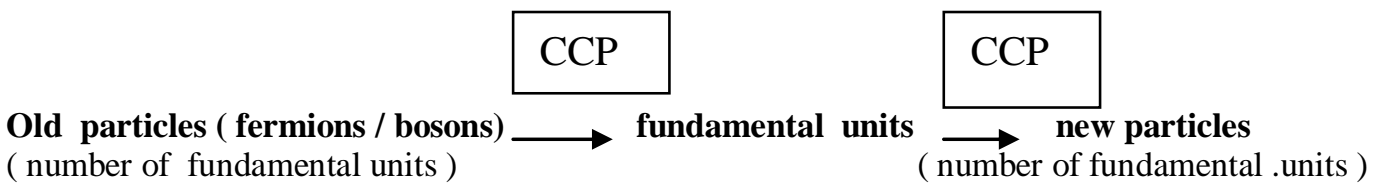

2. Stimulation of thoughts could be conditioned or by unconditioned way ( figure 3 ).

3. The raw stuff that would be required during transformation would come from inside quark (energized gravitons, secondary boson ( binding energy -3 ), from inside energized graviton ( primary boson- i.e. energy pool of universe.).

4. The production of ( excessive energy) dark energy ( inactive form) would go back to energy pool of universe i.e. inside energized gravitons in form of primary bosons. Production of excessive matter or dark matter (inactive form of fermions) would be lost in space. Not visible by any eye.

5. The thought expressions of old particles are suppressed and thought expressions of new created particles are triggered or become dominant. Thus new properties appear in nature .

6. The transformation is so fast that we are unable to see intermediate products ( fundamental units) and we are forced to see few new particles only. Some times we are unable to observe all created particles as they are not in active form ( dark matter ( fermions )and dark energy (bosons). Hence the equation is some times not balanced. We have to make it balanced only when we know the entire structure up to the level of basic building blocks of the old particles and new particles

7. All decay phenomenon of subatomic particles are destined and destiny is triggered by stimulus . Hence it is type of stimulus that decide the MODE of decay. It is called conditioned thought stimulation. Hence low energy decay have different fate while high energy decay have different fate. . Some times fate is also decided by self stimulation or unconditioned way . We could see this phenomenon in creation physics .

\section{Message from biological world as regard}

One sperm and one ova fertilized to form one baby with in nine months . It is a transmutation of sperm and ova into fetus or baby of $3 \mathrm{~kg}$ in weight .

1 sperm ( micro gram) + 1 ova ( micro gram ) $\longrightarrow$ baby ( $3 \mathrm{~kg}$ )

$$
\text { decay }
$$

A physicist would explain the equation that one sperm and one ova annihilate to form a baby of $3 \mathrm{~kg}$. He would not consider that how could microscopic organism having few micro gram weight would decay into 3 $\mathrm{kg}$ baby. It is impossible. But it does happen in nature. This is observatory science .

Participatory science explains that there is a dark matter which is not taken into consideration to get the equation balanced. Now on taking dark matter into consideration, the equation is like this

1 sperm ( few microgram ) + 1 ova ( few microgram ) + Dark matter ( $2.999999 \mathrm{~kg}$ )

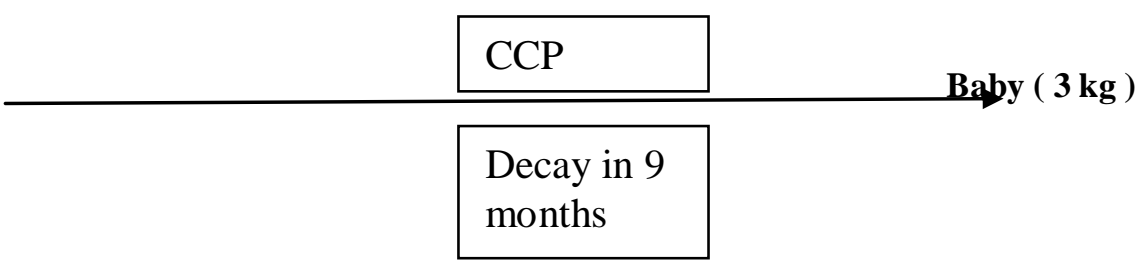

Now the equation is balanced. This dark matter $(2.99999 \mathrm{~kg}$ ) is in form of food taken by mother during 9 months. That helps to make the baby of $3 \mathrm{~kg}$. In this process of decay MIND is involved . Hence CCP is also written. Unless mind triggers , nature cannot decay or transform.

This is the philosophy of decay of every phenomenon in the universe Structure of fermions and bosons of old particles and new particles 


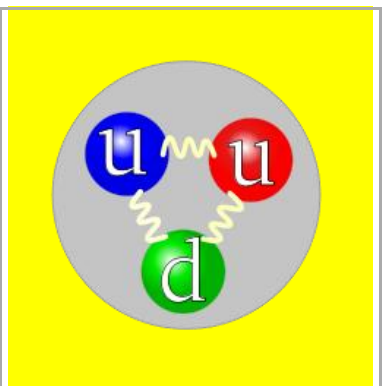

Figure 7 Wrong depiction of proton [3]

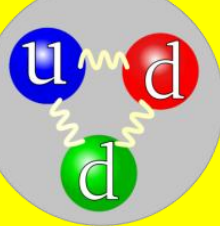

Figure 7.1 wrong depiction of neutron
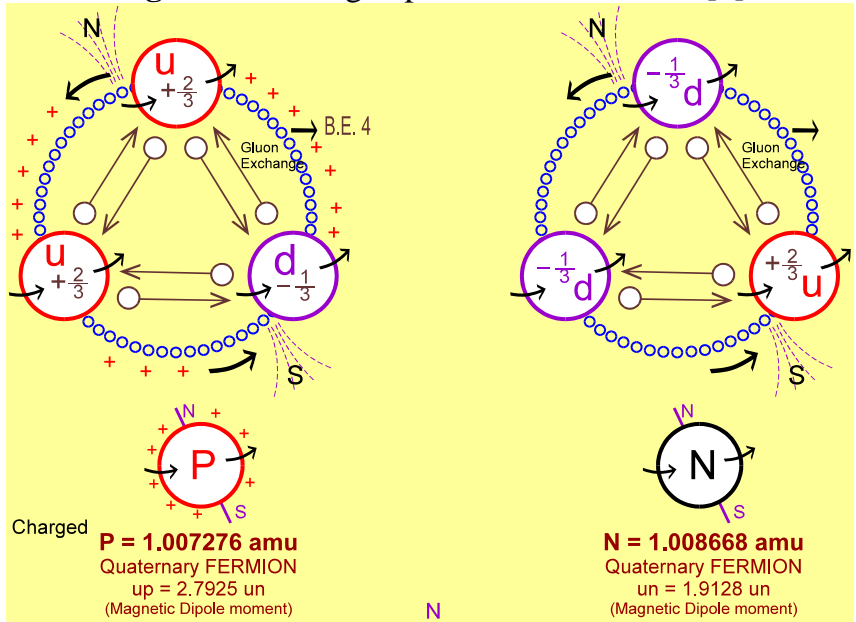

S

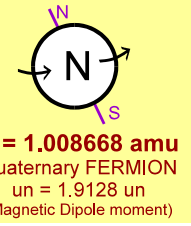

(Figure 7.2 STRUCTURE OF PROTON AND NEUTRON ( B.E.-4 or spin -4))

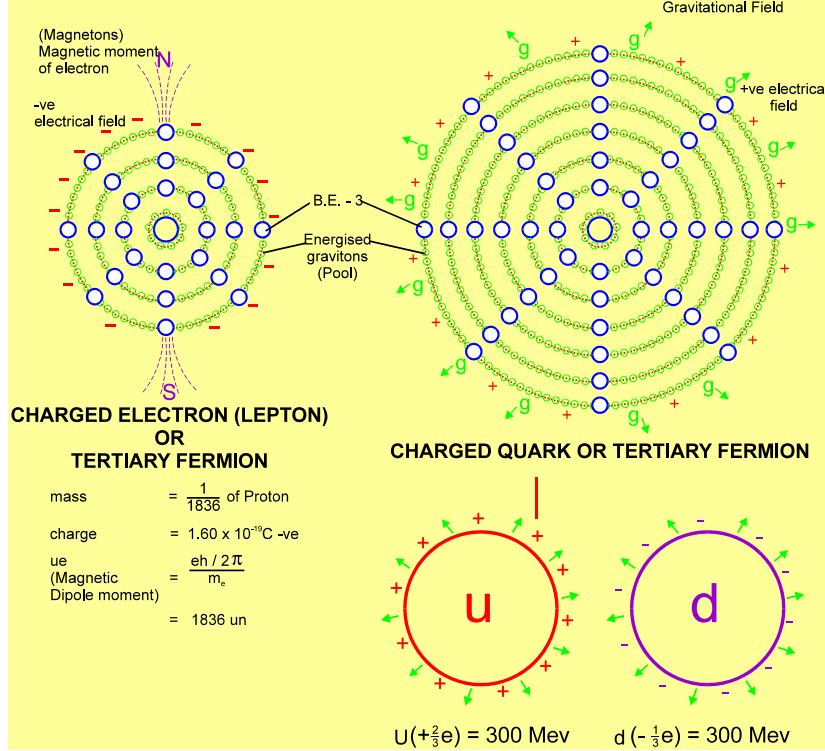

Figure 8 STRUCTURE OF QUARK AND ELELCTRON ( B.E. -3 or spin -3) 


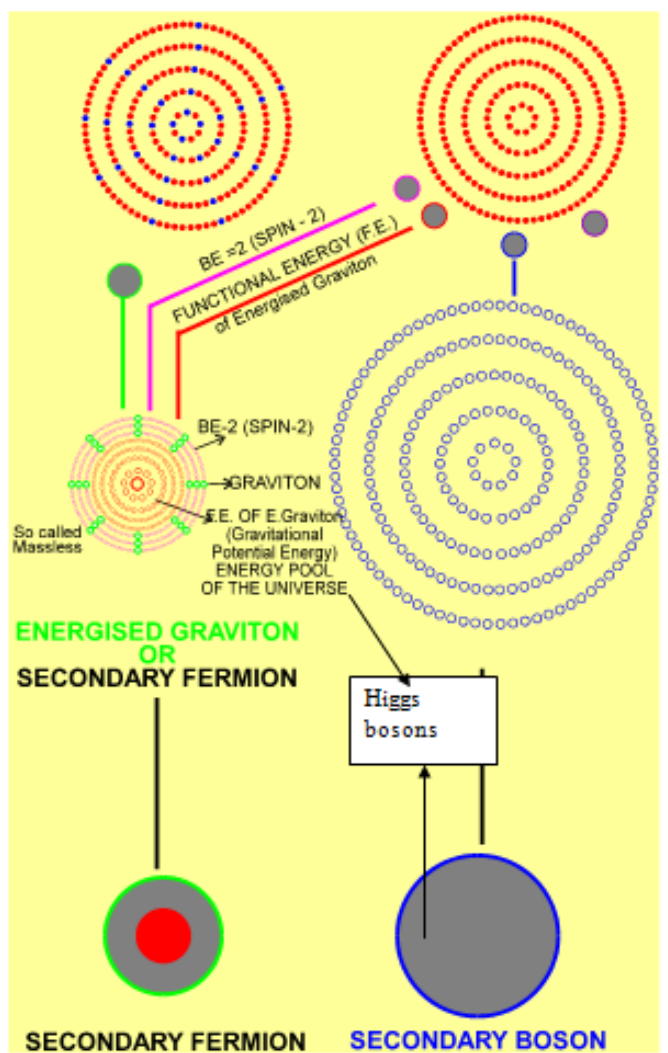

Figure 9 STRUCTURE OF ENERGISED GRAVITONS ( Binding energy -2) or( spin -2) AND SCONDARY BOSONS - HIGG'S (BINDING ENERGY or B.E.)

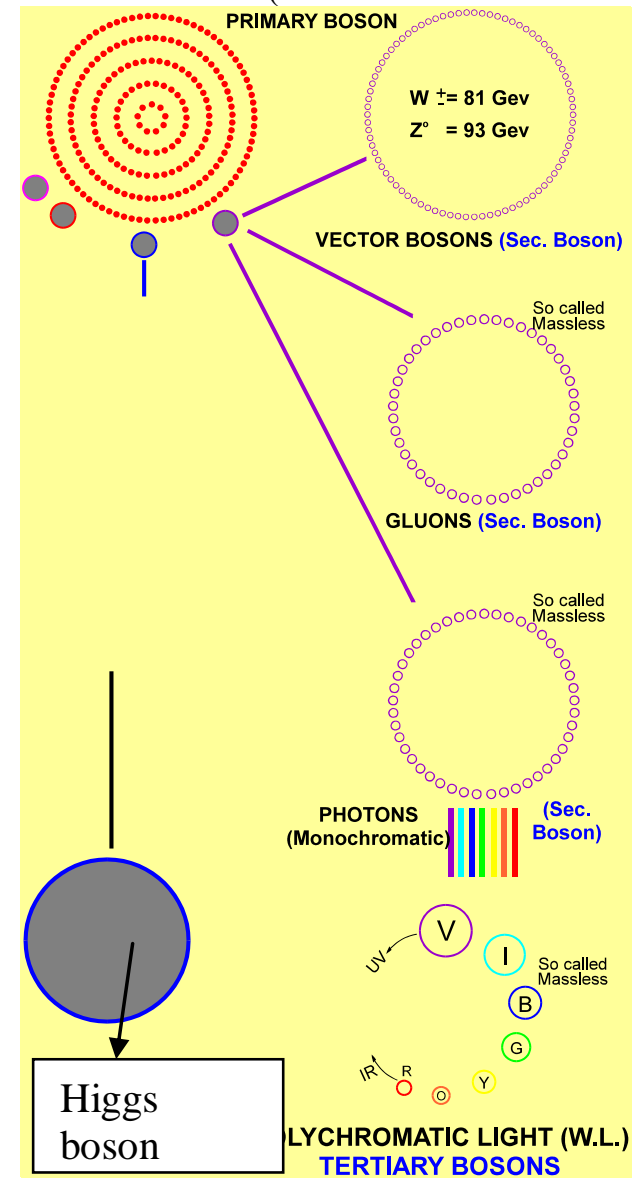

Figure 10 STRUCTURE OF POLYCHROMATIC, MONOCHROMATIC, GLUONS, VECTORBOSONS 


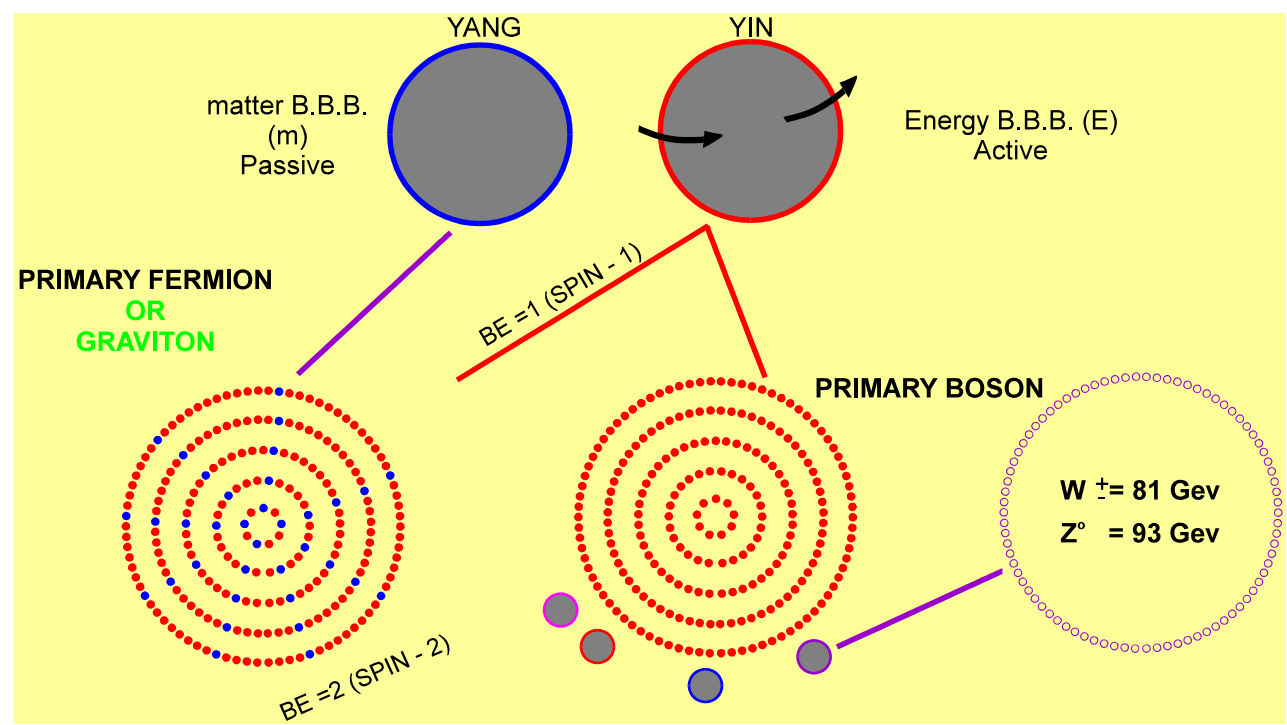

Figure 11 Structure Of Primary Fermions And Primary Bosons ( Binding Energy -1 ( Spin -1)

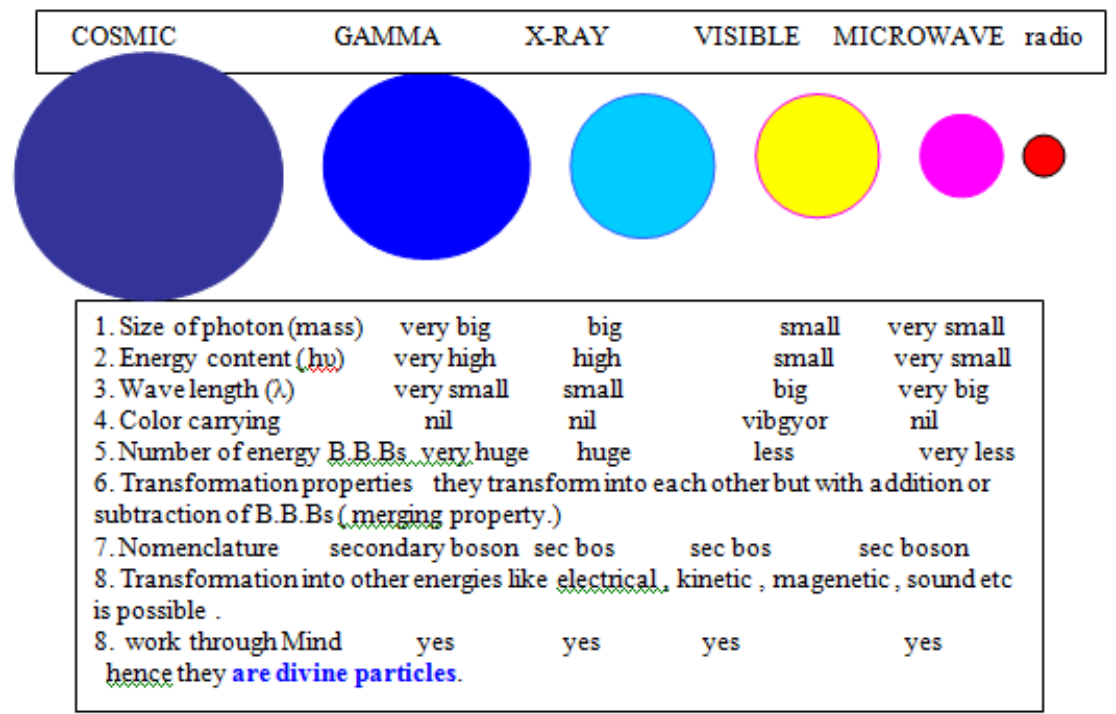

Figure 12 STRUCTURE OF GAMMA RAYS , XRAS , VISIBLE RAYS , MICROWAVE

1. Stability and beta decay ( figure 13 ) [5]

wrong depiction

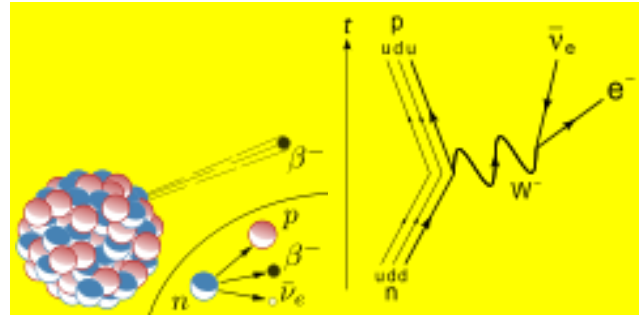

( figure 13 Beta decay [5]) 


\section{RIGHT DEPICTION}

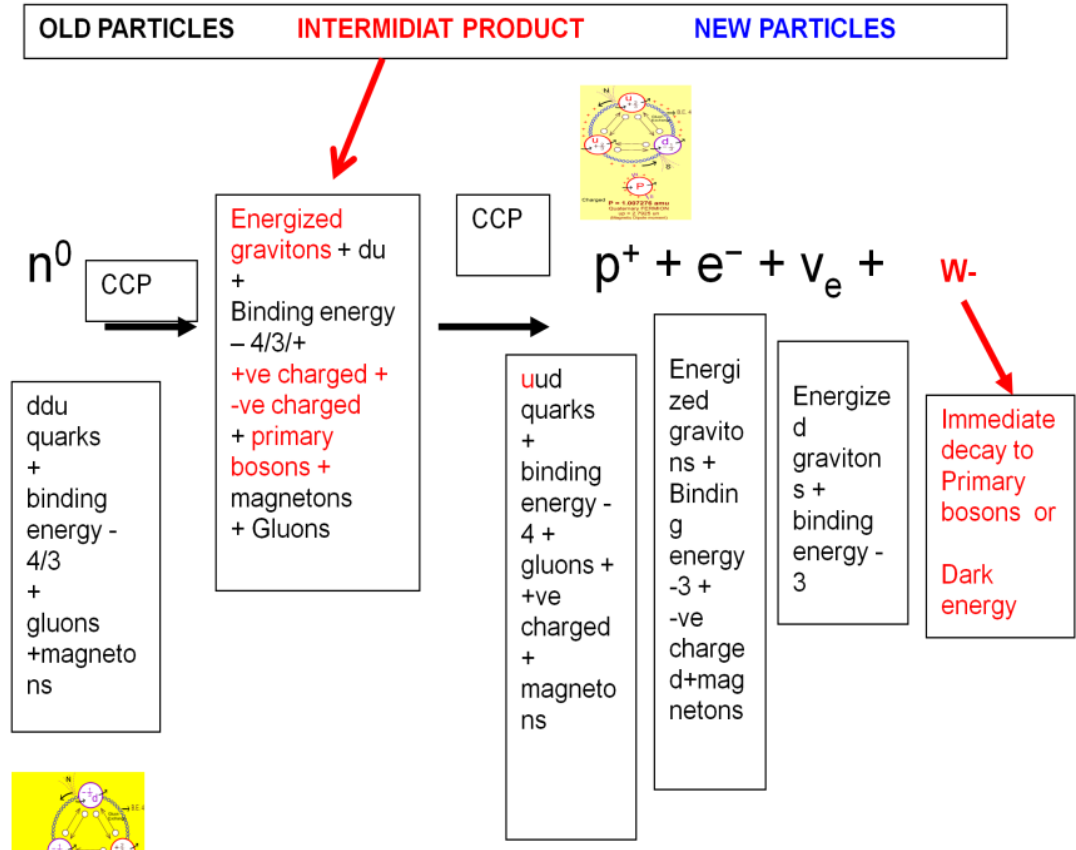

\section{ANNIHILATION [6]}

( figure 14 beta decay )

\section{a. WRONG DEPICTION}

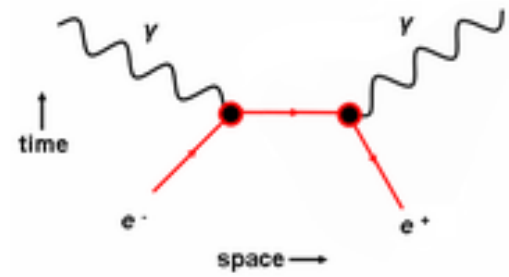

( figure 15 electron positron annihilation [6] )

RIGHT DEPICTION

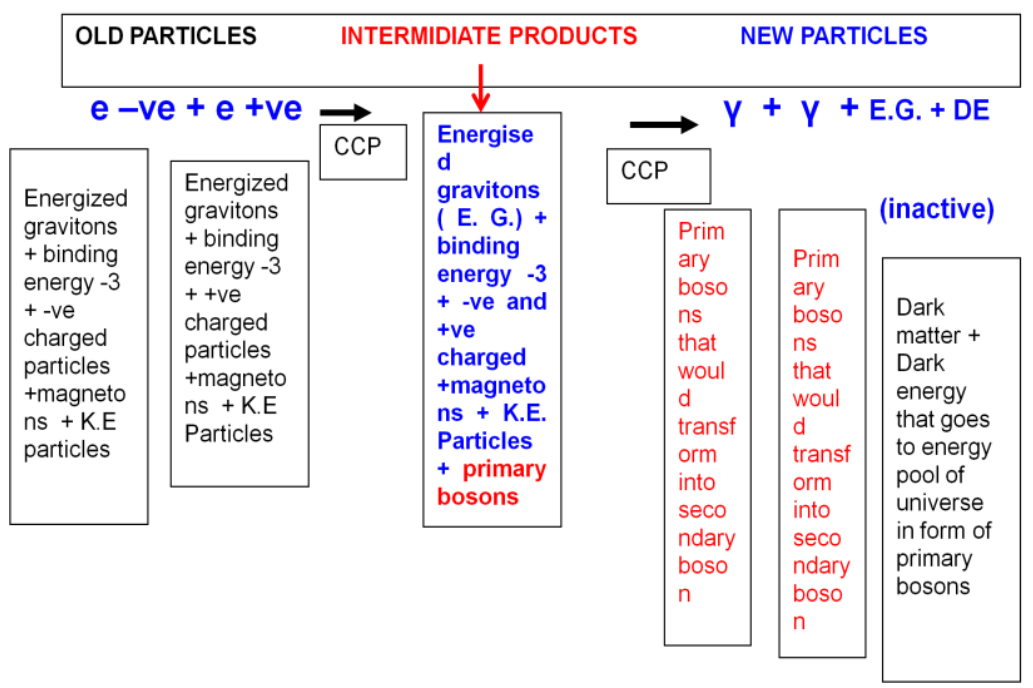

Annihilation

( figure 16 electron positron annihilation ) 
b. WRONG DEPICTION [7]

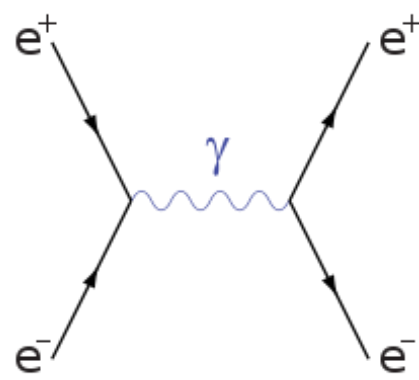

( figure 17 electron positron Annihilation [7] )

RIGHT DEPICTION

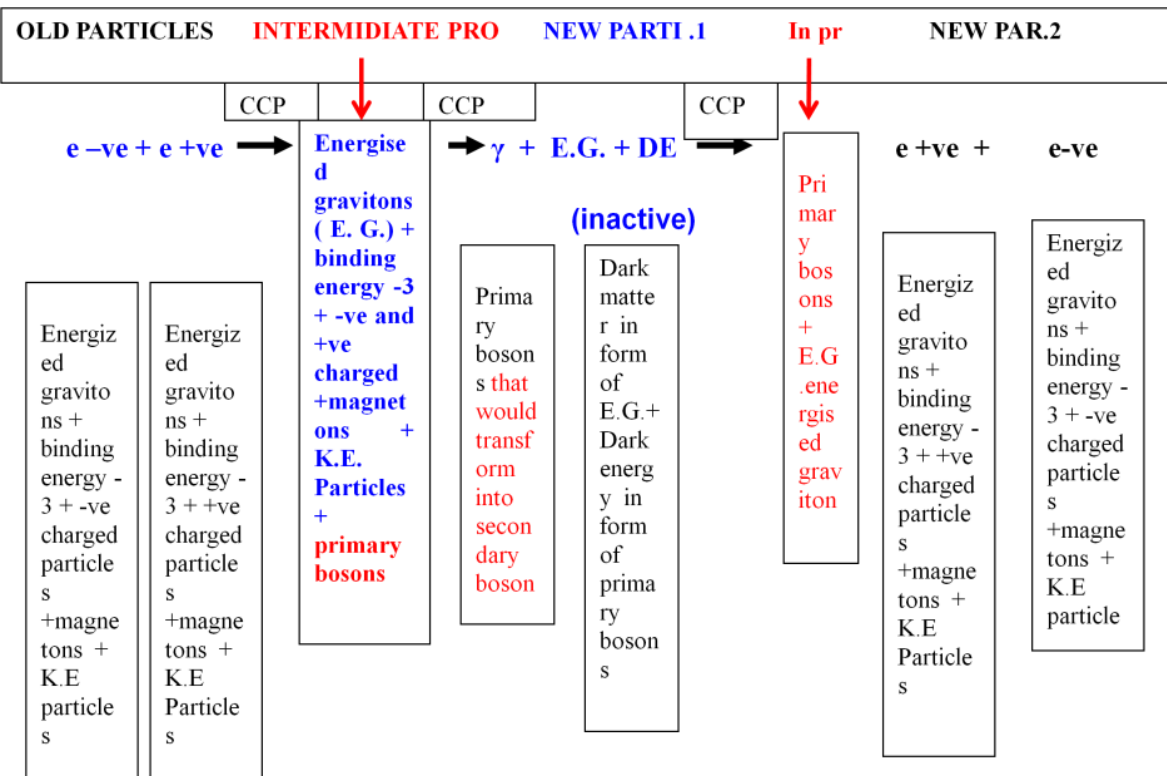

( figure 18 electron positron Annihilation )

Inverse Compton scattering

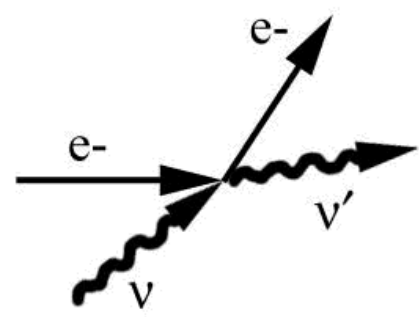

$$
v^{\prime}>v
$$

High energy e- initially

e- loses energy

( figure 19 INVERSE COMPTON SCATTERING) 
RIGHT DIPECTION

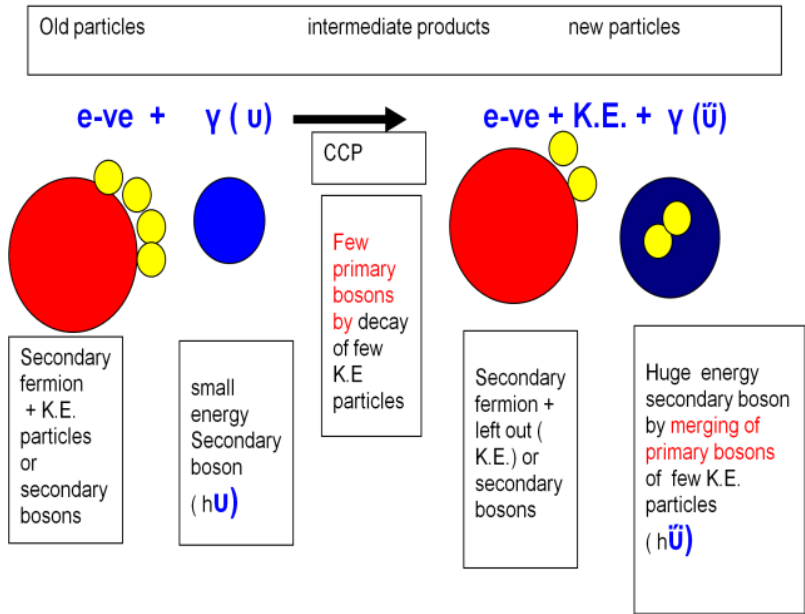

( figure 20 INVERSE COMPTON SCATTERING)

\section{COMPTON EFFECT} WRONG DEPICTION [9]

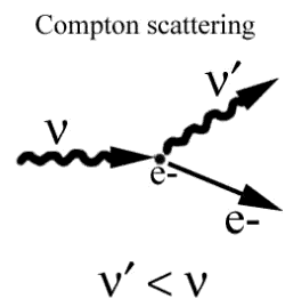

Electron is initially at rest

e- gains energy

( figure 21 Compton effect [9] )

RIGHT DEPICTION

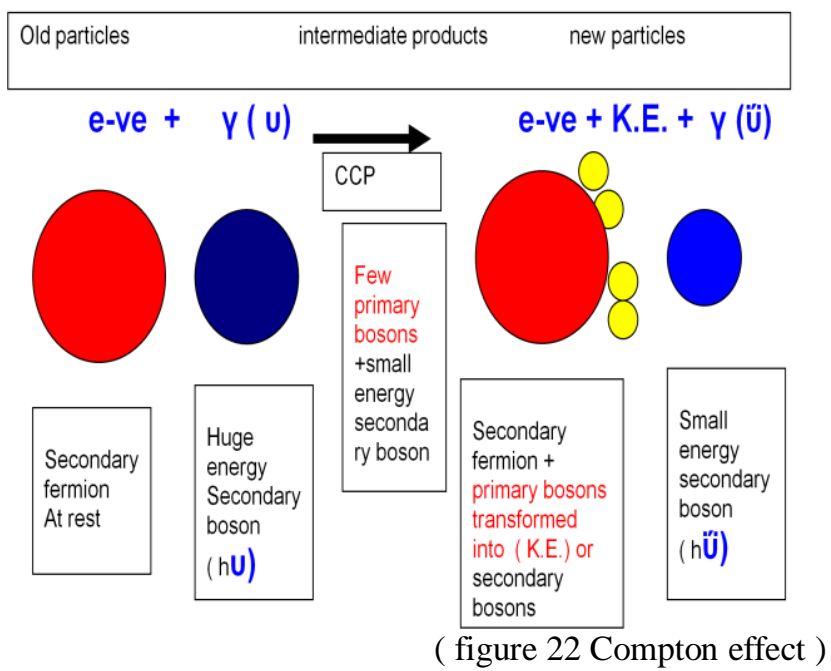

CONCLUSIONS - To understand phenomenon of decay one must have full knowledge of structure of the matter up to the level of basic building blocks, with knowledge of MIND with knowledge to make the equation of decay balanced on right hand and left hand side in terms of fundamental units i.e. of which the older particles and new created particles are made up of and knowledge of dark matter and dark energy i.e. raw stuff that also take part in decay. 


\section{3 spin property of micro particles.}

Before we discuss about spin property and mind, we should know about Basic rotation Rate and classification of particles on spin basis .

\section{BASIC ROTATION RATE}

When we deal with particles at the quantum level, we find that elementary particle can spin only at certain well specified rates. To understand what quantum mechanics tells us about particle spin . we can introduce the concept of BASIC ROTATION RATE. This is the fundamental unit in terms of which the actual spin of the particle is measured. Quantum mechanics tells how to calculate this rate of any particle.

Technically, the basic rotation rate is calculated by setting the angular momentum of the rotating particle equal to planck's constant. For example sphere of mass $\mathrm{M}$ and radius $\mathrm{R}$ turning once every $\mathrm{T}$ seconds, this corresponds to the requirement that $4 \pi \mathrm{MR}^{\wedge} 2 / 5 \mathrm{~T}=\mathrm{h}$

The value of $\mathrm{T}$ obtained from this equation is what we are calling the basic rotation rate.

The proton, for example, has a basic rotation rate of about $10^{\wedge} 22$ complete revolutions per second .

The significance of the basic rotation rate is this ; particles can spin only at rates that are certain well defined multiples of their basic rotation rat. They can for example, spin twice as fast or half as fast, but they can not spin three fourth or two thirds as fast. In the language of quantum mechanics, we can say that spin of the particles is quantized.

For the proton the number is $1 / 2$. In the other words, we find experimentally that the proton spins at precisely half of the basic rotation rate that the quantum mechanics assigns to it. In the jargon of high energy physics, we say that the proton has spin $1 / 2$ or it is a spin $1 / 2$ particle. Particles that rotate exactly at the basic rotation rate are said to have spin-1. Particle that do not rotate at all have spin -0, and so on .ANY particle we talk about will have some rotation rate, and this rotation rate is constant in time.

\section{CLASSIFICATION BY SPIN}

Particles that have half -integer spin $-1 / 2,3 / 2,5 / 2$, and so non , are called fermions, after the physicist Enrico Fermi.

Particles that have integer spin $-0,1,2$, and so on - are called bosons, after Indian physicist S.N. Bose .The only boson we have encountered so far is the photon, the particle associated with electromagnetic radiation.

Fermions and bosons are truly distinct classes of particles in the sense that no interaction has ever been seen that can convert one in to other. This distinction, as we shall see, persists back to the earliest stages of Big Bang.

( The moment of creation - pages 55-58 by James S . Trefil )

After seeing the structure of particles up to the level of basic building blocks .( see Figs below), cause and effect concept and unconditioned thought stimulation, we understand spin phenomenon.

\section{LAWS TO REGULATE SPIN PROPERTY OF PARTICLES}

The spin property of particles is triggered by thought of higher center present in that particles. Thought of half integer led to half inter spin and thought of integer spin led to integer spin property of the particle . It is due to unconditioned thought stimulation. Hence the spin property is constant in time. No external influence could change it . It is the numerical mindness that controls Basic rotation rate of all particles. Spin and it is the numerical mind ness thought that triggers spin of above mentioned objects. These are fed thoughts and these were fed during precreation era by highest center of universe to B.B.Bs which are now working as higher center in particles, nucleus of atoms, planets, satellites, suns and galaxies .

The spin is induced by Binding energies ( B.E.-1,2,3,4, and 5). B.E.-1 to 4 for different particles and B.E -5 for nucleus of atom. Till these B.E. are there, these structures would keep on spinning. Planets, satellites and suns are being rotated ( SPIN) by rotational energies being supplied by energized gravitons to them from energy pool of universe. The same energy pool of the universe maintain charge property and magnetic property of particles, planets, suns, etc. These are fed thoughts and these were fed during precreation era by highest center of universe to B.B.Bs which are now working as higher center in particles, nucleus of atoms, planets , satellites, suns and galaxies .

Hence spin is basically a MIND and Mass ( energy mass and matter mass ) interaction. Mind is numerical mindness thought expression and direction mindness thought expression that controls rate of spin and direction ( clock or anti clock wise ) of spin. Mass ( energy mass and matter mass for fermions and only energy mass for bosons ), that occupies space moves in spin direction. 


\section{WHY DO PARTICLES , NUCLEUS OF ATOMS , PLANETS, SETALLITES, SUNS AND GALAXIES} SPIN ?

Particles do spin because -

1. It is the property of fermions and bosons and nucleus of atoms.

2. By B.E. - 1,2,3, and 4, primary, secondary, tertiary and quaternary fermions get spin. And primary and secondary bosons get spin.

3. By B.E.-5, nucleus of atoms get spin.

4. Spin makes matter B.B.B into spin motion ( primary fermion i.e. graviton) .

5. Spin makes secondary fermions, tertiary fermions and quaternary fermions into spin motion.

6. Spin make primary bosons into spin motion .

7. Spin makes secondary bosons into spin motion.

8. Spin makes nucleus of Atom in to spin motion.

9. These spin motions help to quantize the particles from one another.

10. Spin helps in shifting the messages ( interaction part) from one part of atoms to another part of atom.

11. Gravity interaction is totally based on spin of objects and spin of energized gravitons . Starting from bending of starlight to planetary motions of planets etc. Had there been NO spin , no gravity effect is visible . ( $\mathrm{pl}$ read gravity chapter.)

12. Gravity is not exchange phenomenon. It does not show shielding effect and it is due to interaction between energized gravitons and spin of nucleus of atoms.

13. We measure gravitational mass in amu (atomic mass unit) of particles and nucleus and it could only be possible due to spin property of nucleons and nucleus . Pl. see mass defect and energy release from fusion and fission.

14. All effects like Raman effect, Zeeman effect, VIBGYOR formation is due to spin property of electron that moves around nucleus in atom.

15. All scientific interactions in physics, chemistry and biology are due to electron spin motion.

16. Planets, satellites, asteroids and galaxies do spin because

a. For our planet - During formation of earth from rotating nebula, earth got separated only when spin got triggered. . Hence spin makes formation of celestial bodies from hydrogen and helium cloud.

b. For our moon - do

c. For our sun - do

d. For asteroids- do

e. For galaxies - do - GMC transformed into bright galaxy only when spin of proto star triggers . Spin of galaxies makes these suns into orbital motion around the center of mass of galaxies by gravity interactions. The stars nearer move faster than those which are away from center.

CHARGE PROPERTY OF PARTICLES AND NUCLEUS OF ATOMS Particles are charged as they help in making afferent paths (-ve charged particles photons from electron to nucleus ) and efferent paths ( + ve charged particles photons from nucleus to electrons ) for messages transaction ( second transcription ) from atom to atom.

MAGNETIC PROPERTY OF PARTICLES AND NUCLEUS AND PLANET

Will be discussed later on

GRAVITY PROPERTY OF PARTICLES, NUCLEUS AND PLANET etc

Pl see gravity chapter ( Volume 4 part 1 and part 2 )

CLASIFICATION BASED ON SPIN PROPERTY.

ELEMENTARY PARTICLES---- Classification of fermions and bosons based on their spin properties is a wrong classification. The classification must be according to their constituents i.e. types of BASIC BUILDING BLOCKS (B.B.Bs) of which they are made up of . According to participatory science gravitons are fermions but they have integer spin. On this basis super symmetry i.e. symmetry between fermions and bosons could never be achieved. That is why supper unification could never be proved as B.B.Bs i.e. matter B.B.B and energy B.B.B are separate and they can never be transformed into one another being opposite in properties. (eternal properties or inertial properties of energy and matter ).

You did not talk about research of B.B.Bs in your booklet. Without this research, high energy physics can never be understood correctly. It is the basic research that will help us to know about the truth as regard origin of the universe, ,origin of earth, and solar system, origin of life and all mysteries of physics and life sciences. It will produce fragrance of GOD in models of the origin of the universe etc. Mathematics alone cannot produce thought of God in all physical models.

THE UNIFICATION OF PHYSICS----- GUT and super unification ( one particle theory) have not proven yet. Symmetry between fermions and bosons are not proven yet. Big Bang model does not have fragrance of God. One cannot create conditions of Big bang in lab. The entire Big Bang physics is hypothetical. On the 
contrary my two-particle theory has been proved successfully. There are observations that confirm two particles hypothesis.

Evidences

Fermions and bosons are truly distinct classes of particles in the sense that no interaction has ever been seen that can convert one in to other. This distinction, as we shall see, persists back to the earliest stages of Big Bang. [10]

UNCERTAINTY PRINCIPLE-- The famous metaphor said by Einstein that God does not play dice has ultimately become the truth . His dream became successful with the research of basic building blocks and atomic genetics. Our universe is basically deterministic universe and deterministic orders of the universe are governed by ATOMIC GENES or thought statements. All effect is triggered by thought statement or atomic transcription. Being observer we cannot predict the nature precisely. Had we been Participator (highest center of the universe, or basic building blocks of the event), we could have precisely predicted the event. The theory predicts that when highest center ( B.B.B working as highest center) would shift to earth from invisible universe, all un-predictable events of quantum physics would be predicted precisely and with certainty. Our universe follows the laws of atomic genetics and all thoughts are fed thoughts and that too were fed by highest center of the universe during precreation era. So there is no question of uncertainty in their precise prediction by the participator or by highest center of the universe (ALMIGHTY OR GOD).

2.4 Faster than Light - phenomenon of Prayer and phenomenon of Pre-creation era that led to origin of the universe

when photon was not created i.e. fundamental interaction among God particles To understand faster than light phenomenon ( FTL), we should know Message system of the universe. ( Figure -23)

\section{Message system of the Universe}

Before the origin of the universe nature had only one type of message systems which is called FIRST TRANSCRIPTION. Messages used to go from one B.B.B. to another B.B.B. by atomic transcription. Messages were carried by atomic genes with very very high velocity. It is the fundamental message system.

After the origin of the universe, nature created atoms. It also created one more message system called SECOND TRANSCRIPTION. Here the message (code Pcps) are carried by photons from one atom to another atom with velocity of light. Thus atoms , molecules, cells, and even individuals talk with one another

After the formation of the cell, nature created one more system called THIRD TRANSCRIPTION .Here there is a message storage system formed by DNA. There are messenger molecules called mRNA that carry message from DNA script to cytoplasm where the message (code PCPs) is read or translated by ribosome and they work accordingly. Thus the messages reach to enzymes and hormones and finally messages reach to target units. Having received the messages, target units work accordingly. Finally life effects (metabolic) are observed. These three types of message systems are working in the nature. These message systems are being used by the nature according to nature's need. ( Fig-23) 


\section{MESSAGE SYSTEMS OF THE UNIVERSE}

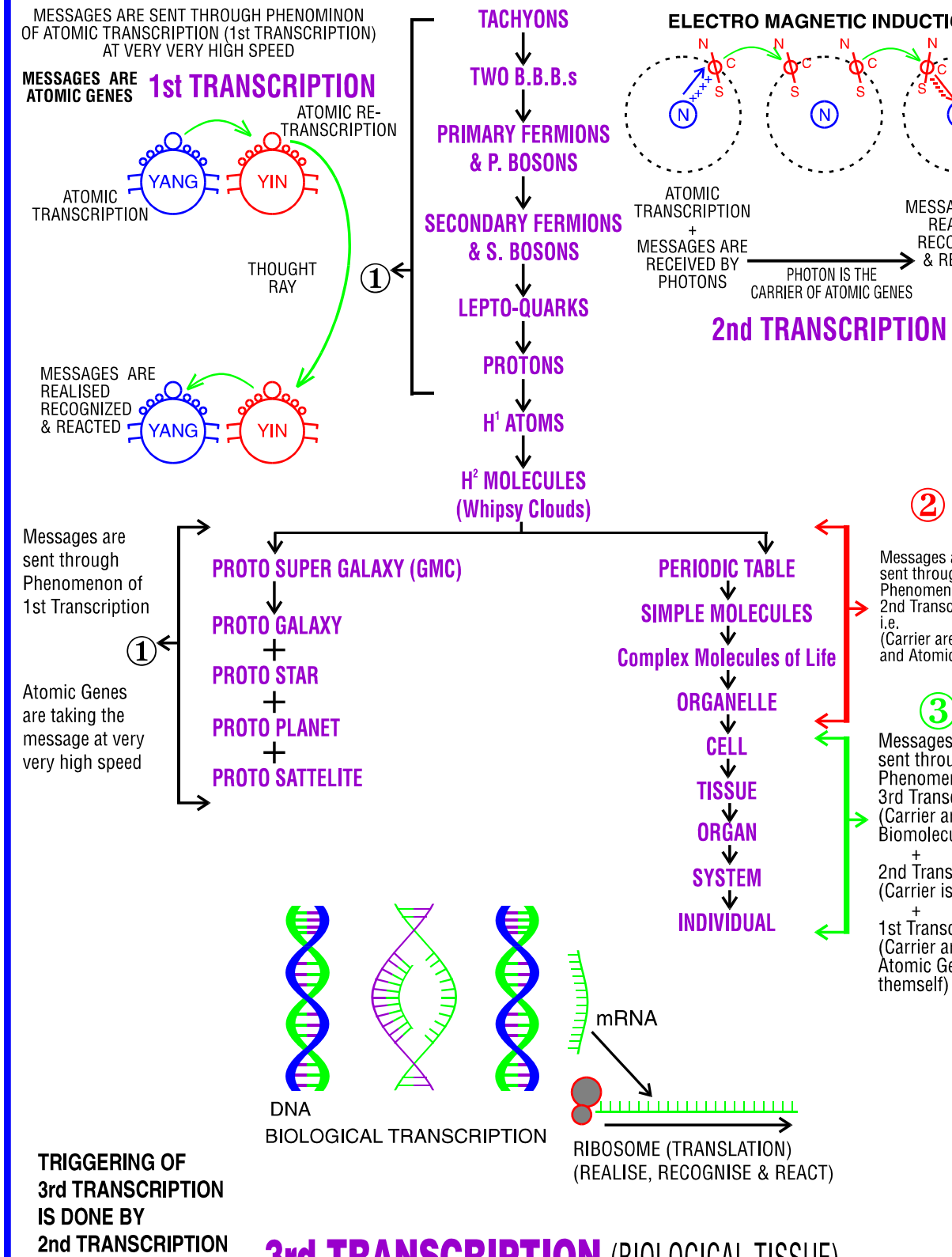

DNA

BIOLOGICAL TRANSCRIPTION

2nd TRANSCRIPTION

(2) 
atom is made up of B.B.Bs. At the level of B.B.B. say thought of 'O GOD HELP ME' is expressed. As a result programmed messages of O GOD HELP ME (code PCPs) are formed. Out of three programmed messages, one is carried by atomic genes to highest center of the universe. It is called THOUGHT RAY which is made up of pure atomic genes and then the message goes through phenomenon called first transcription. They come out from brain directly. The other two messages are carried by photons from nucleus of atom to electrons. Here they are modulated on electrical activity of the cell called pacemaker activity. Further they are modulated on actions potentials going towards REALIZING CENTER situated in brain stem (RAS) and from RAS to speech area situated in the frontal area. Target B.B.Bs. of the realizing center finally realizes thought effect of O GOD HELP ME. While from speech area message goes to motor cortex again via RAS and from there to vocal cords and finally it comes out as a speech effect of O GOD HELP ME. In layman's terminology formation of the thought ray means PRAYER. The details would be given in next section of brain and atomic genetics along with the other mysteries of the brain.

The message goes to highest center of the universe where it is realized and it is accepted, the highest center sends two messages to B.B.Bs. working as higher center in cancer cell. These messages are message of inhibition of abnormal thought expression and message of activation of normal thought expression. Having received the messages, higher center stops expressing the abnormal thoughts and it starts expressing the normal thoughts. As a result, there are no more abnormal programmed messages and in place of that normal programmed messages are there. Now the messages have shifted from abnormal (iii \&iv) to normal (i \& ii). This shifting of thought expression is called ATOMIC GENETIC ENGINEERING ( Figure 26). The changed messages reach to target B.B.Bs. through same route. Having received the changed messages, target B.B.Bs. stop expressing the previous programming and they start expressing the normal programming. As a result the cancer cells transmutate into normal cells. Or diseased cell gets cured.

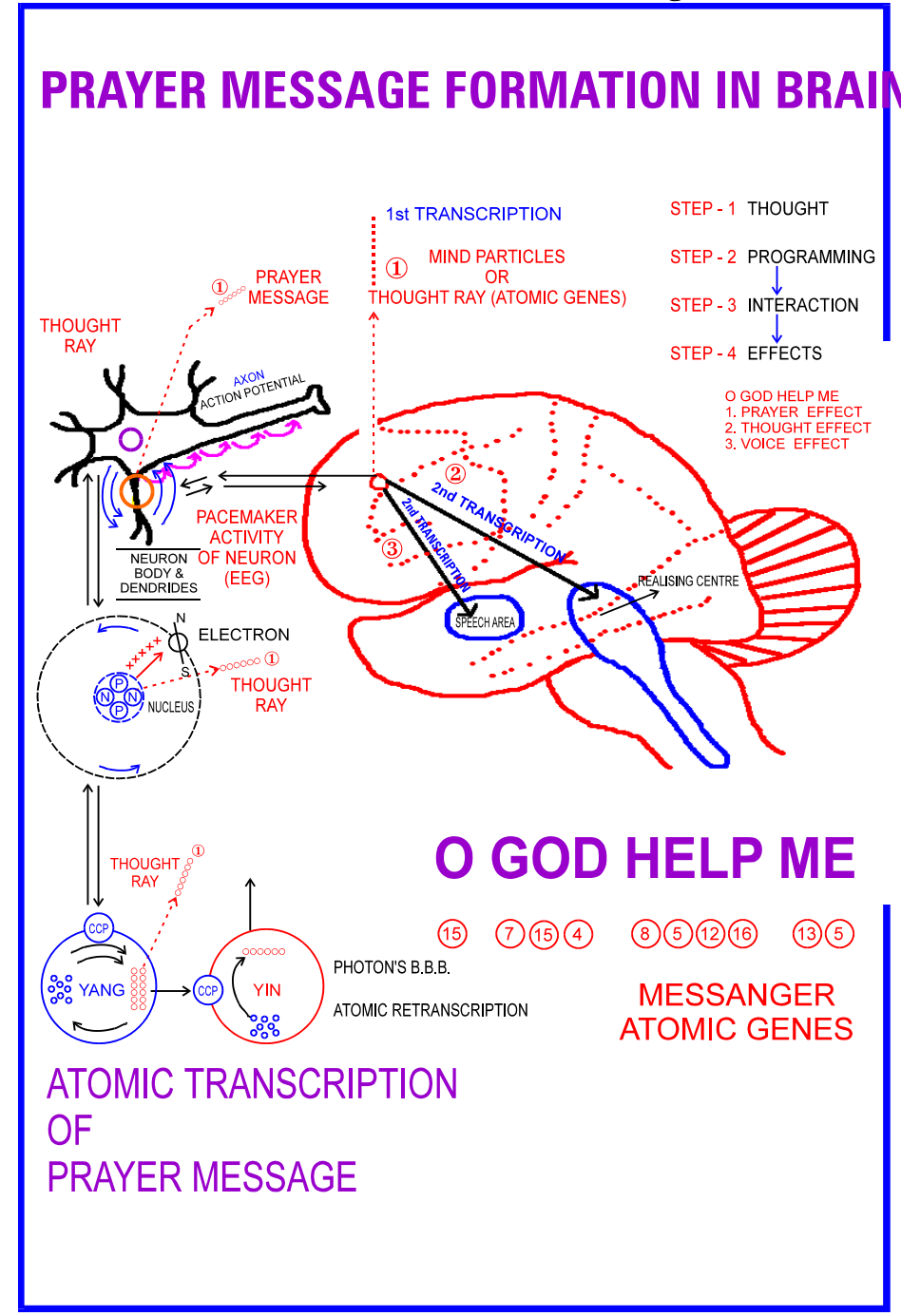

( Fig-24)

\section{Message network of the Universe}


With the origin of universe, nature first created primary units i.e. primary fermions (gravitation) and primary boson, these primary units are equipped with one higher center (one B.B.B.) and rest of the B.B.Bs. are working as lower centers or target B.B.Bs.(A). After primary units ,nature created secondary units i.e. secondary fermions and secondary bosons. similarly nature created tertiary units (lepto-quarks) and then quaternary units (protons\& neutrons).

Each unit is equipped with higher centers, lower centers and target B.B.Bs. After quaternary units nature created atomic units, molecular units, complex molecules of life units, organelle units, cell units, tissue units, organ units, system units and individual units. Each unit is equipped wit higher centers, lower centers, and target B.B.Bs. Similarly nature created satellite units, planet units, solar system units, galaxy units, super galaxy units, dark matter layer unit. These units are also equipped with higher centers, lower centers and target B.B.Bs.

Thus our universe is divided into different units and each unit is equipped with higher and lower centers. All higher centers are under control of highest center of the universe by efferent paths. This efferent path is made up of first transcription. Higher centers can send messages to highest center of the universe by afferent path or feed back path. Thus highest center of the universe is well informed about all effects of the universe. Messages can come from lower centers to higher centers and from higher centers to highest center of the universe via afferent path. The highest center of the universe can send messages to higher centers and from higher center to lower centers. There is an inter unit message network also which is made up of first, second and third transcription depending upon the nature's need. Thus the entire universe is under control of highest center of the universe. Highest center can change any programming programmed by it during pre creation era. ( Figure25)

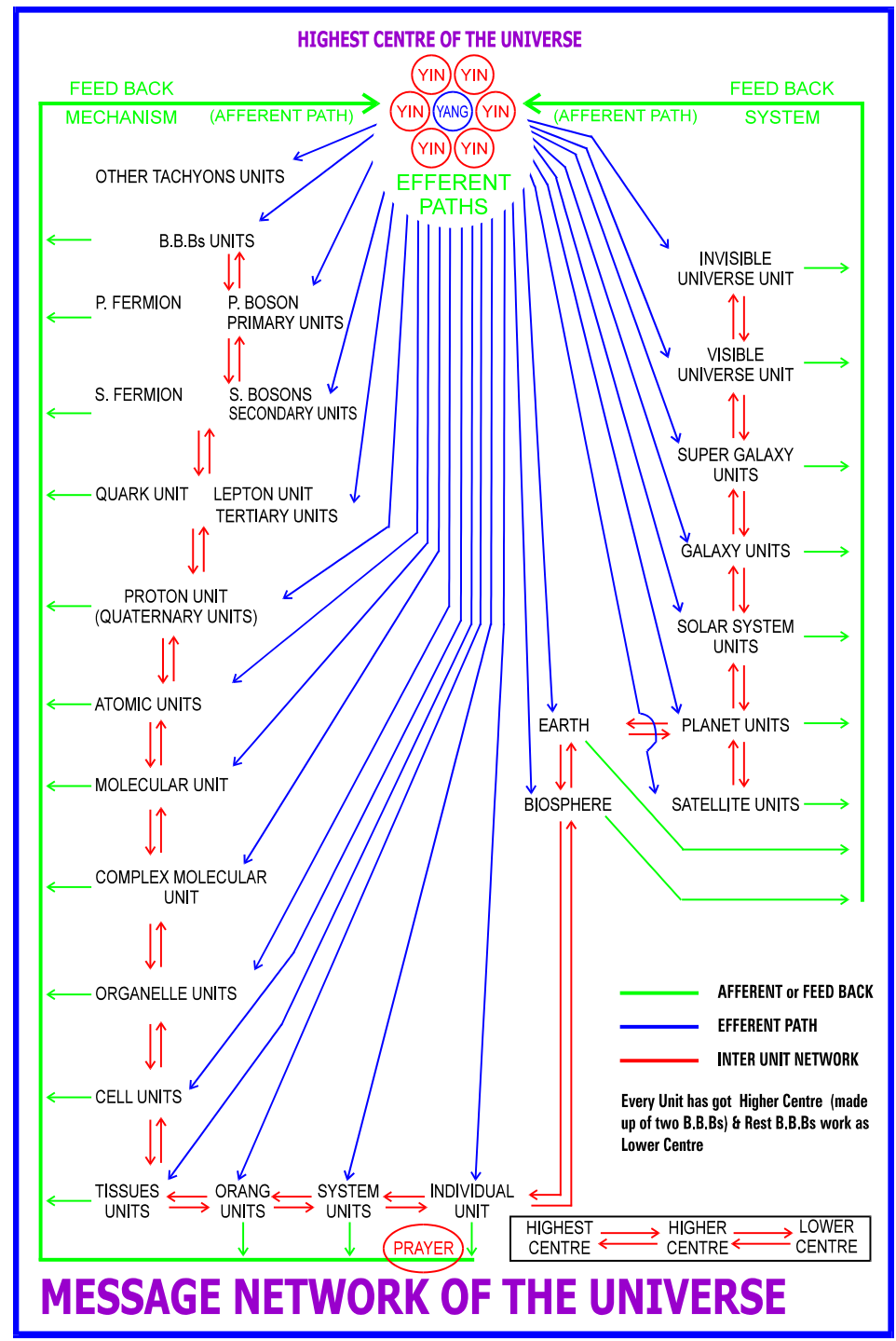

( Fig-25)

ATOMIC GENETIC ENGINEERING (Fig-26) The message goes to highest center of the universe where it is realized and it is accepted, the highest center sends two messages to B.B.Bs. working as higher center in 
cancer cell. These messages are message of inhibition of abnormal thought expression and message of activation of normal thought expression. Having received the messages, higher center stops expressing the abnormal thoughts and it starts expressing the normal thoughts. As a result, there are no more abnormal programmed messages and in place of that normal programmed messages are there. Now the messages have shifted from abnormal $(4,5,6)$ to normal $(1,2,3$,$) . This shifting of thought expression is called ATOMIC GENETIC$ ENGINEERING. The changed messages reach to target B.B.Bs. through same route. Having received the changed messages, target B.B.Bs. stop expressing the previous programming and they start expressing the normal programming. As a result the cancer cells transmutate into normal cells. See Figure -26

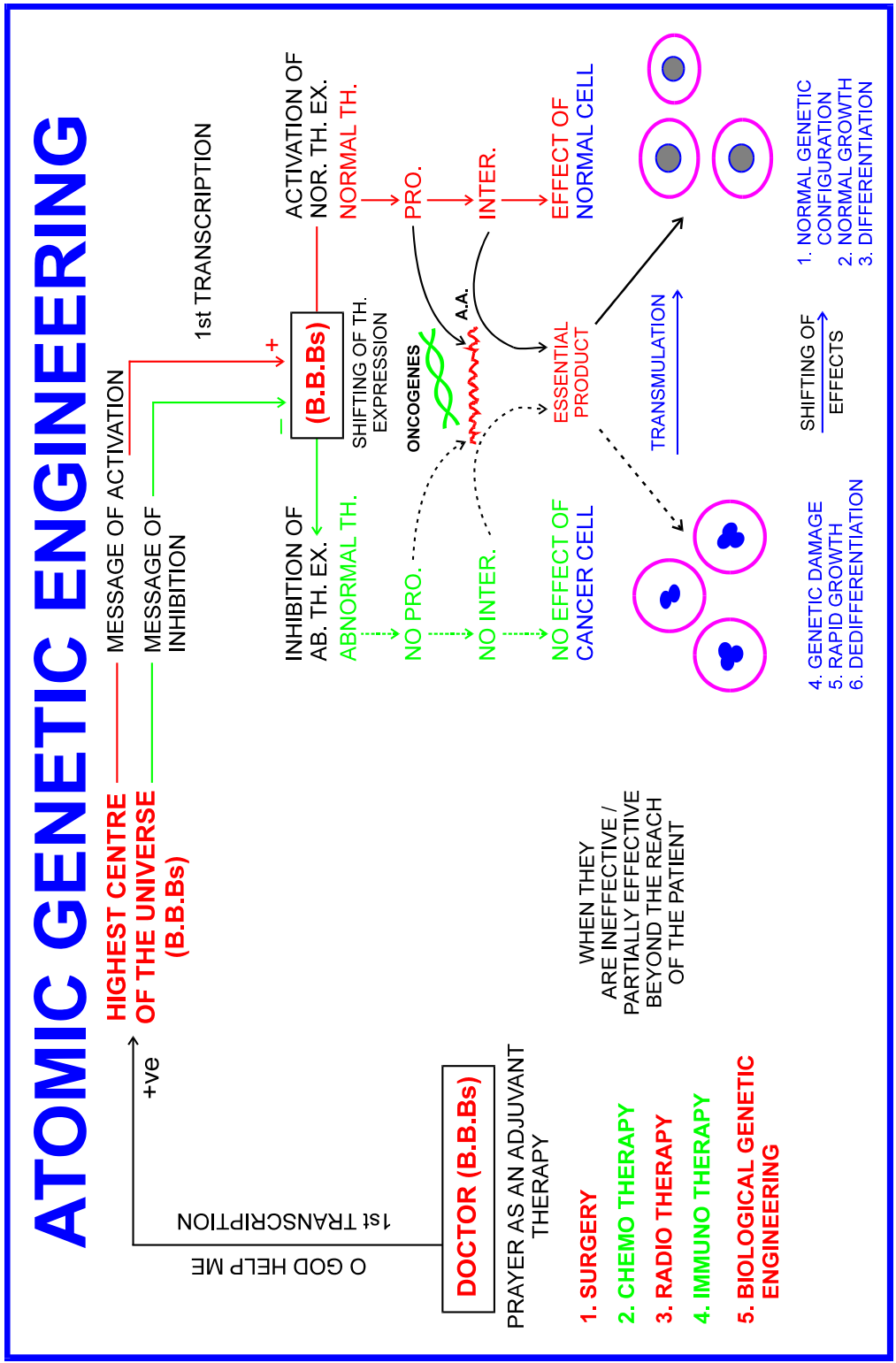

( Fig-26) 
Observation of the Hypothesis

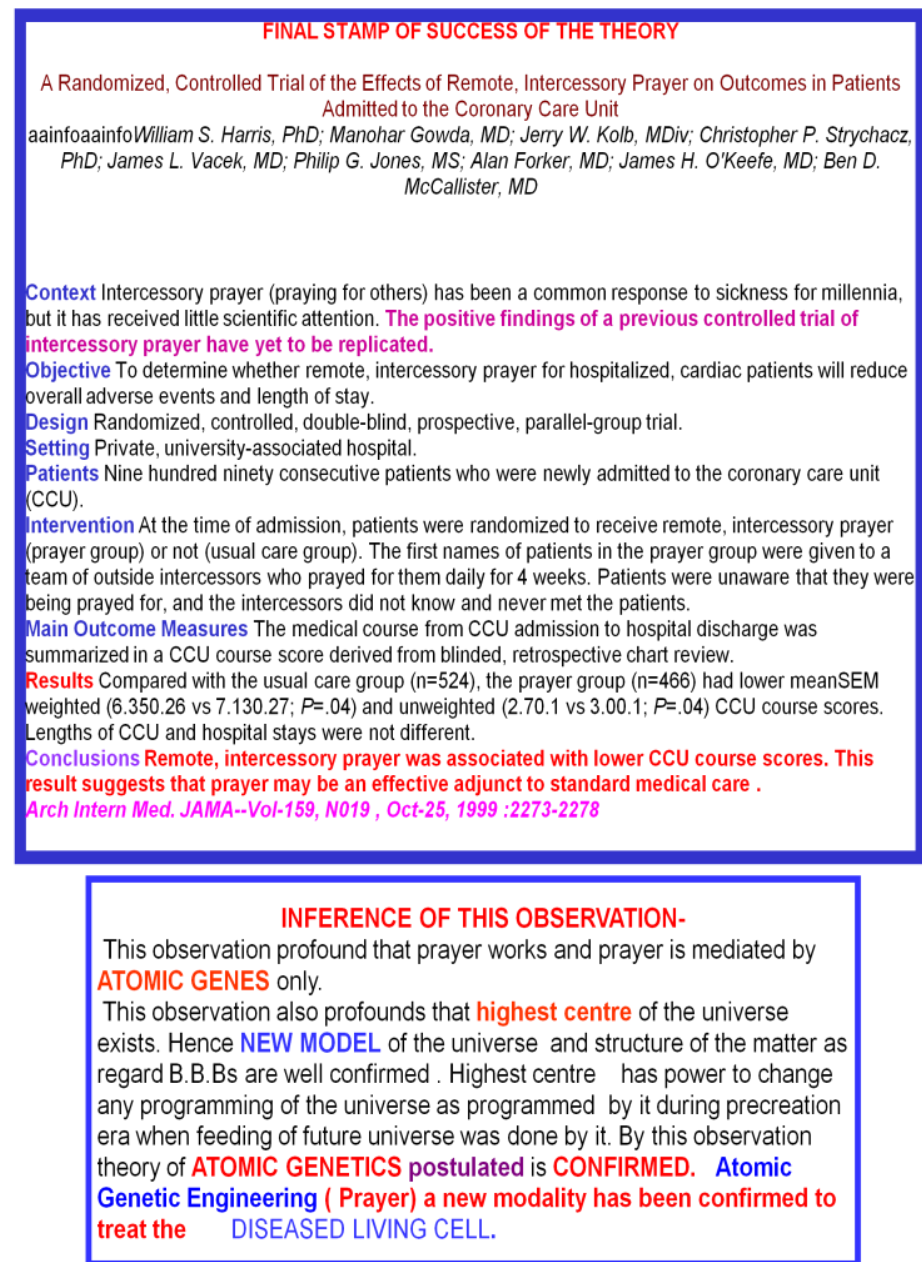

( figure 27 observation of the hypothesis)

Development of the Universe ( Figure- 28)

Before the origin of the universe, these B.B.Bs. were in the form of tachyons. It does mean that the tachyons were every where in the universe. Let us look at the structure of tachyon, it is made up of one of matter B.B.B. (YANG) and many energy (YINs). Initially out of the infinite tachyons, one became the highest center of the universe. Messages used to go from highest center to rest of the universe and messages could come from rest of the universe to highest center of the universe by atomic transcription. Thus highest center had fed its thoughts to rest of the B.B.Bs. that would take part in creation - that they would express only those thoughts to give desired effect as wished by the highest center of the universe. So all B.B.Bs were informed about their role before creation of the universe. In pre-creation era programming of the future universe was done by highest center of the universe.

\section{( It is faster than light phenomenon that led to formation of our universe.) See Figure.28 ( 1)}

Our universe is oscillating and it is a divine universe. It means that it has a creation phase and a destruction phase. During creation phase tachyons break into their B.B.Bs. and from these B.B.Bs, formation of fermions and bosons take place. After the creation phase, destruction would start and in this phase all created particles would again break into their B.B.Bs. and finally tachyons would form.

At the time of origin of the universe, all the effects got created. These effects are taking of different shapes and appearance of properties and law. Both these effects are studied in different branches of science. The cause of all the effects is THOUGHT. It does mean that unless the thought is expressed, programming is done, the messages reach to target B.B.Bs., nature cannot take new shape, properties and laws change in shape, properties and laws is called TRANSMUTATION. During transmutation target B.B.Bs. show synchronized working of mind and mass. With the result nature takes new shape, properties and laws.

Thought (atomic transcription), programming (formation of messages), interaction (spread of massage) take place on B.B.Bs.. Therefore it is not visible to us. Only the effect part is visible or observed. In each and 
every transmutation, thought expression (atomic transcription) is the essential step. Thought, programming and interaction are collectively called as CCP .So in each step of transmutation, CCP is written. It does mean that unless the thought is expressed, nature cannot transmutated.

Nature takes shape due to mass property of the B.B.Bs.. While appearance of properties and laws are due to different types of thought statements or atomic transcription and which is due to atomic genes. The details shall be presented in chapter of ATOMIC GENETICS

With the origin of the universe, nature first created a sphere of COLD DARK MATTER (C.D.M) and canals in it. With the result space got created. At the other end of the canals, hot reaction started. As a result hydrogen clouds and lot of radiation were created. The empty canals were filled by these hydrogen clouds and radiations and thus QUASARS appeared in the universe. Simultaneously C.D.M. layer started expanding and clouds and radiations kept on coming in this closed universe. With the passage of time more and more C.D.M. layer formed, more and more quasars formed. The hydrogen cloud came in this closed universe. They started running towards C.D.M. layer as they were attracted by the gravity of C.D.M. layer. The HUBBLE LAW can thus be explained (Figure.28(ii,iii \& iv)

With some more passage of time, clouds were joined to form GMC (giant molecular clouds). Later by self gravitation different proto stars, proto planets, proto satellites were formed. Finally stars became bright and thus bright galaxies appeared in this universe. Our universe is still in expansion phase and creation is still going inside quasars. It is to be remembered that highest center of the universe does not come in the visible universe. It keeps on receiving the messages by atomic transcription and it has power to change any programming programmed by during pre-creation era. (Figure-28.)

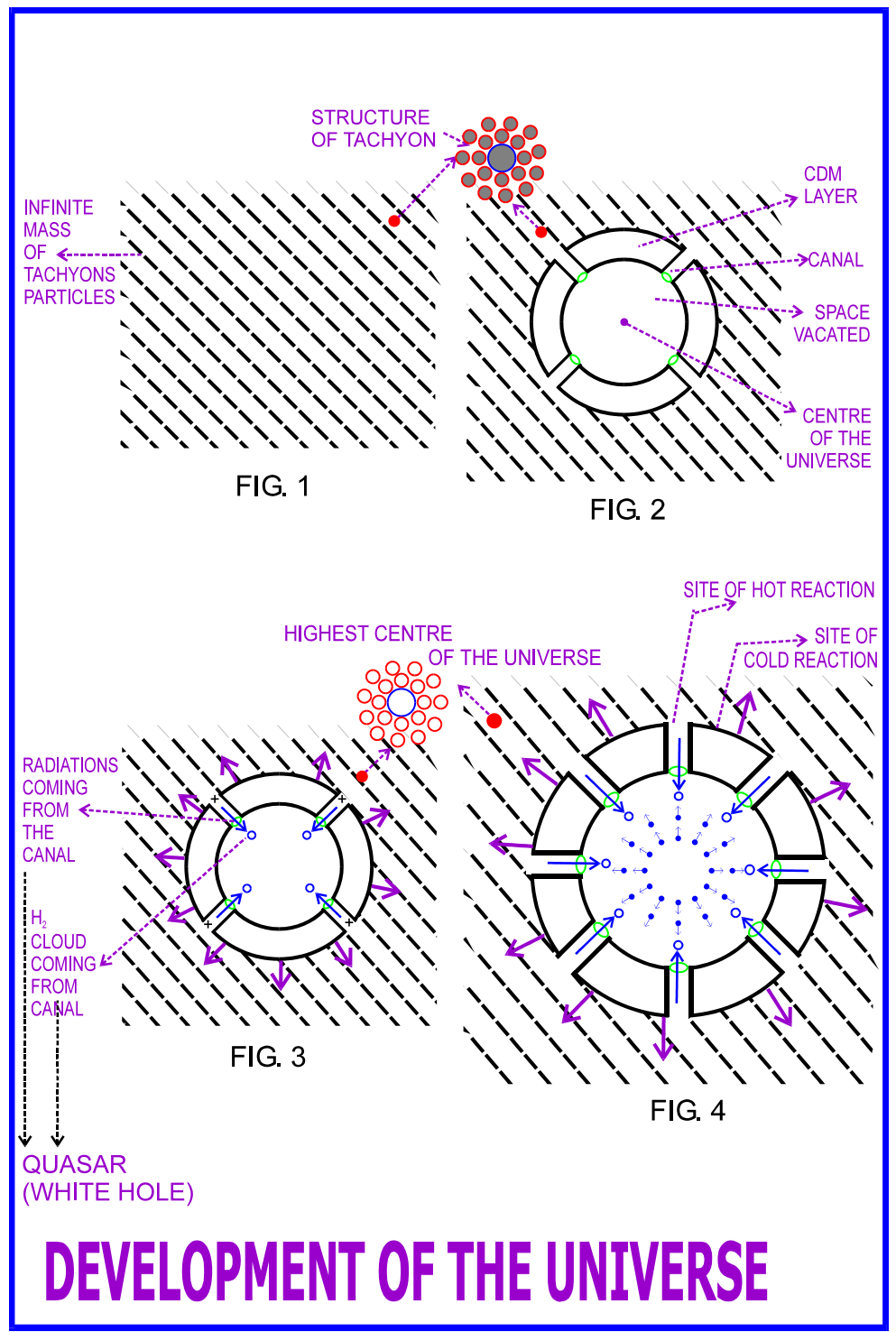

( Fig -28 Development of the universe ) 
2.5 Energy matter interaction ---Transformation of photon into Kinetic energy ( Compton effect ) electrical energy ( photoelectric effect ) and interaction with nucleus ( pair production )

COMPTON EFFECT - WRONG DIPICTION [11]

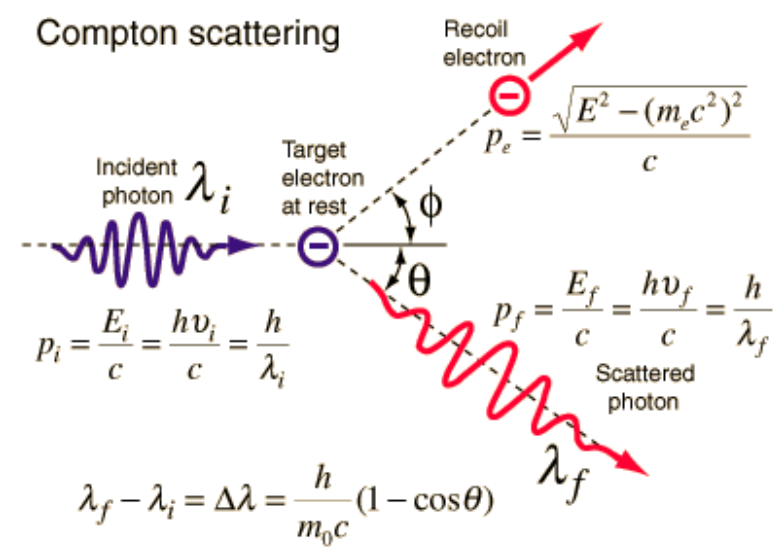

( figure 29 Compton effect [11])

PHOTOELECTRIC EFFECT WRONG DIPICTION [12]

Photoelectric Effect
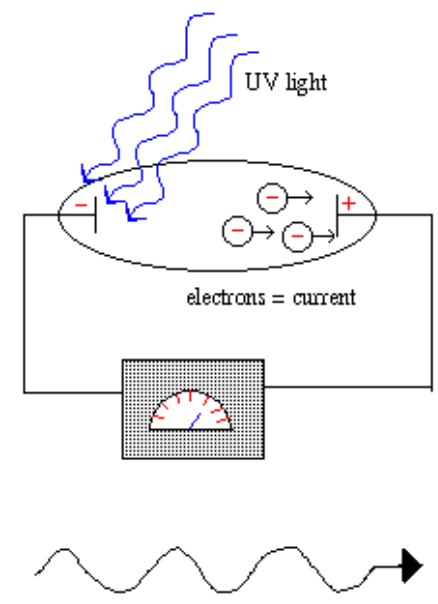

photon $=$ wave particle of light

Figure 30 - ELECTRON=CURRENT

PAIR PRODUCTION WRONG DIPICTION [13]

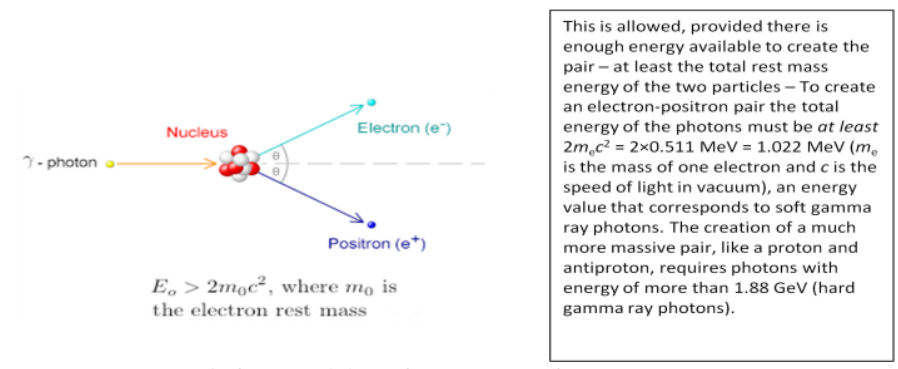

(Figure 31 Pair production [13]) 
CONVENTION - It is believed that current is nothing but streams of moving electrons .It isillusion.

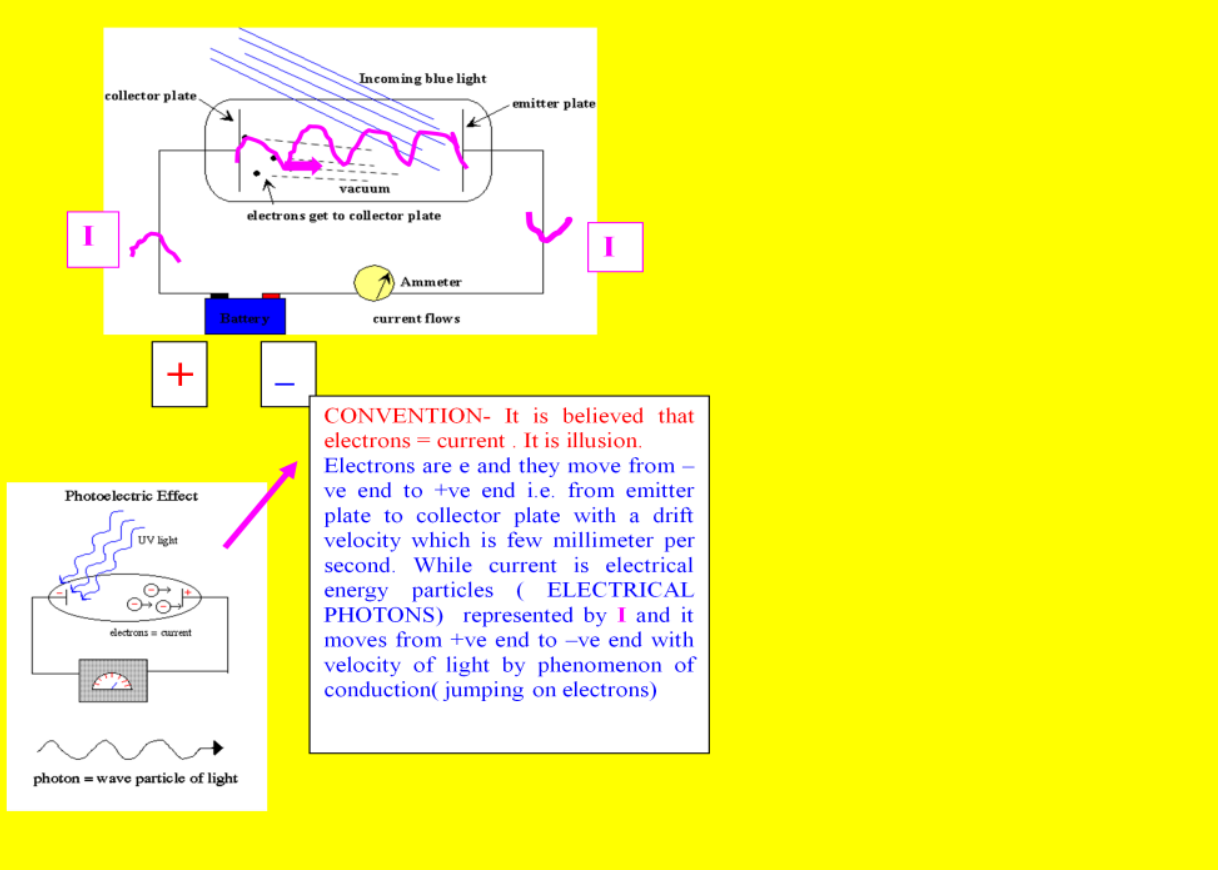

Figure 32 photoelectric effect [14]

Before we understand these phenomena, let us know the structure of electrons, nucleus and photons up to the level of basic building blocks. Diagrams are self explanatory. (Fig-7.2, Figure8, Figure 9, figure10, figure11 and figure 12 )

\section{LET US SEE THEIR DIGRAMATIC REPRESENTATION .}

\section{PHOTOELECTRICEFFECT}

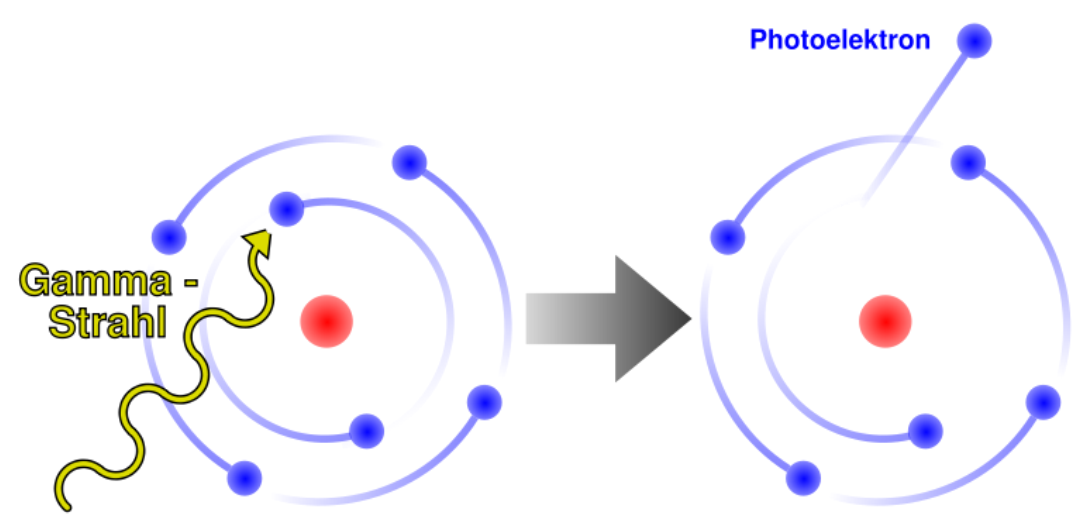

Effect

1. electron is ejected moves from - ve to +ve poles with drift velocity

2. electric current is generated moves from + ve to _ve pole with velocity of light

( figure 33 photo electric effect [15])

\section{COMPTON EFFECT}




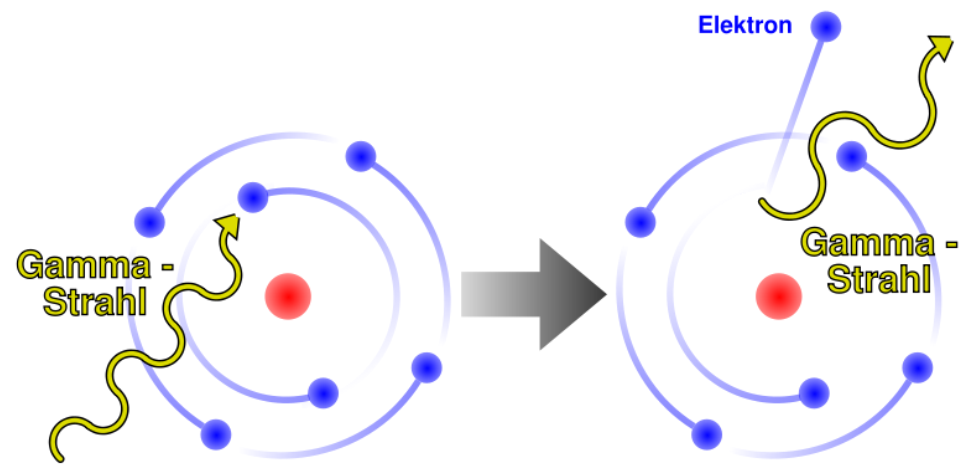

\section{Effects}

1. electron is ejected

2. photon of lower frequency is ejected

( figure 34 compton effect [ 16]

\section{PAIR PRODUCTION}

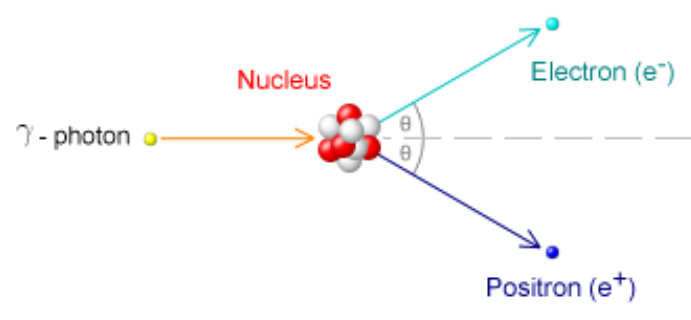

Effect

1. one electron and one positron is ejected

$E_{o}>2 m_{0} c^{2}$, where $m_{0}$ is

the electron rest mass

( figure 35 pair production [17] )

\section{THOUGHTS THAT TRIGGER THESE PHENOMENA}

\section{.WORKING OF ATOMS}

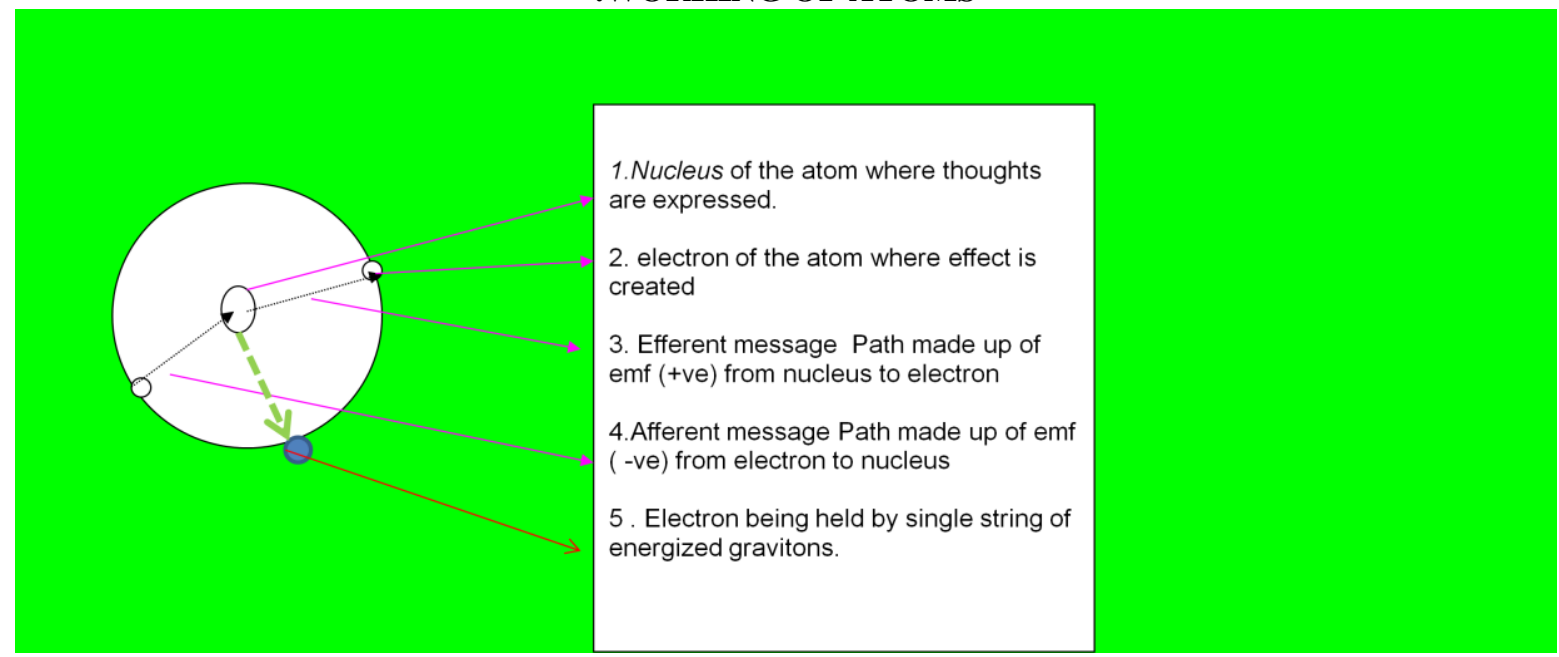

( figure 36 working of atom )

\section{The triggering mechanism of ANY EFFECT is thought}

\section{PHOTOELECTRICEFFECT}

1. Thought of transformation of light energy into electrical energy.

2. Thought of transformation of light energy into kinetic energy of electron to eject electron 


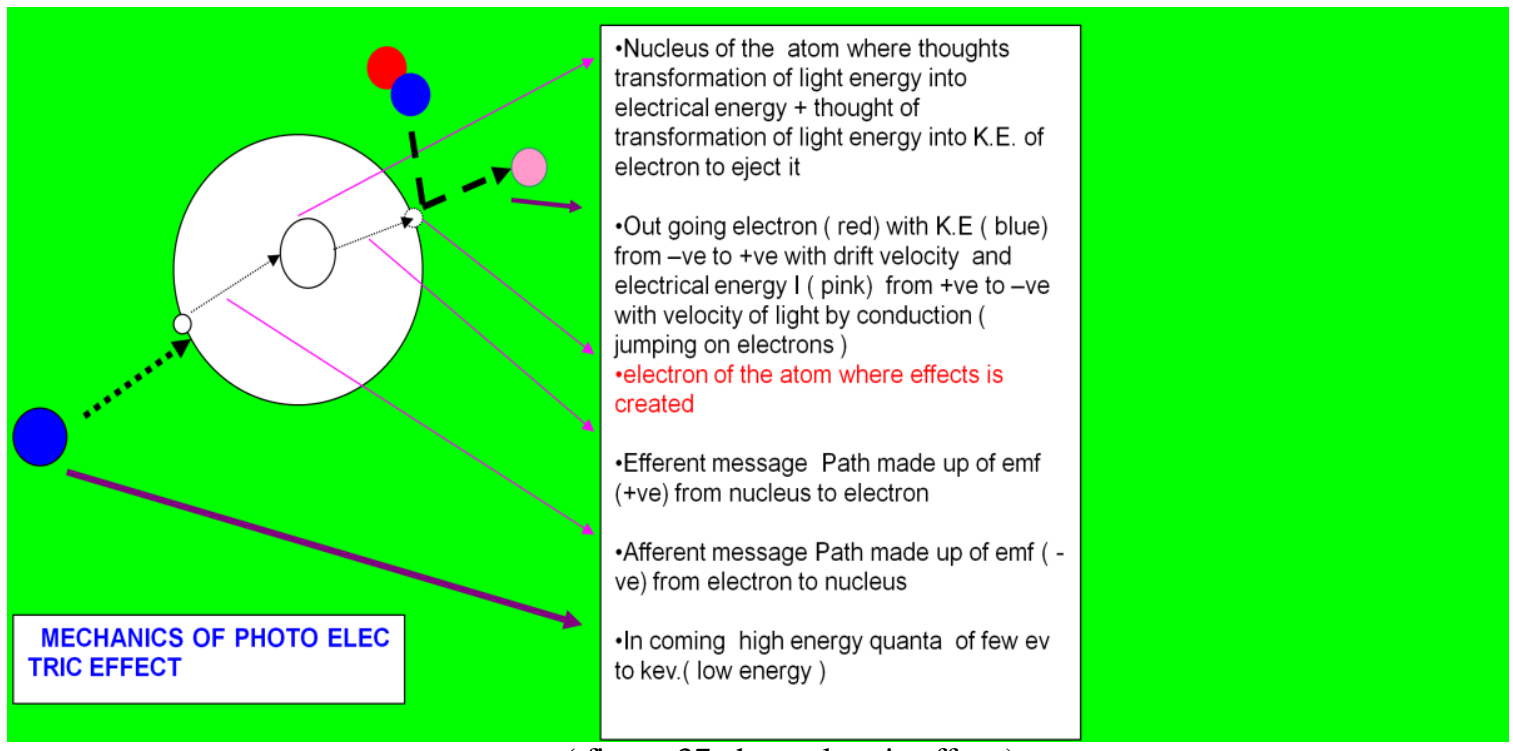

( figure 37 photo electric effect )

\section{COMPTON EFFECT}

1. Thought of transformation of some light energy( higher energy quanta) into kinetic energy to eject electron .

2. Thought of transformation of left light energy into lower energy quanta .

3. Thought of deviation of electron and low energy quanta into different directions

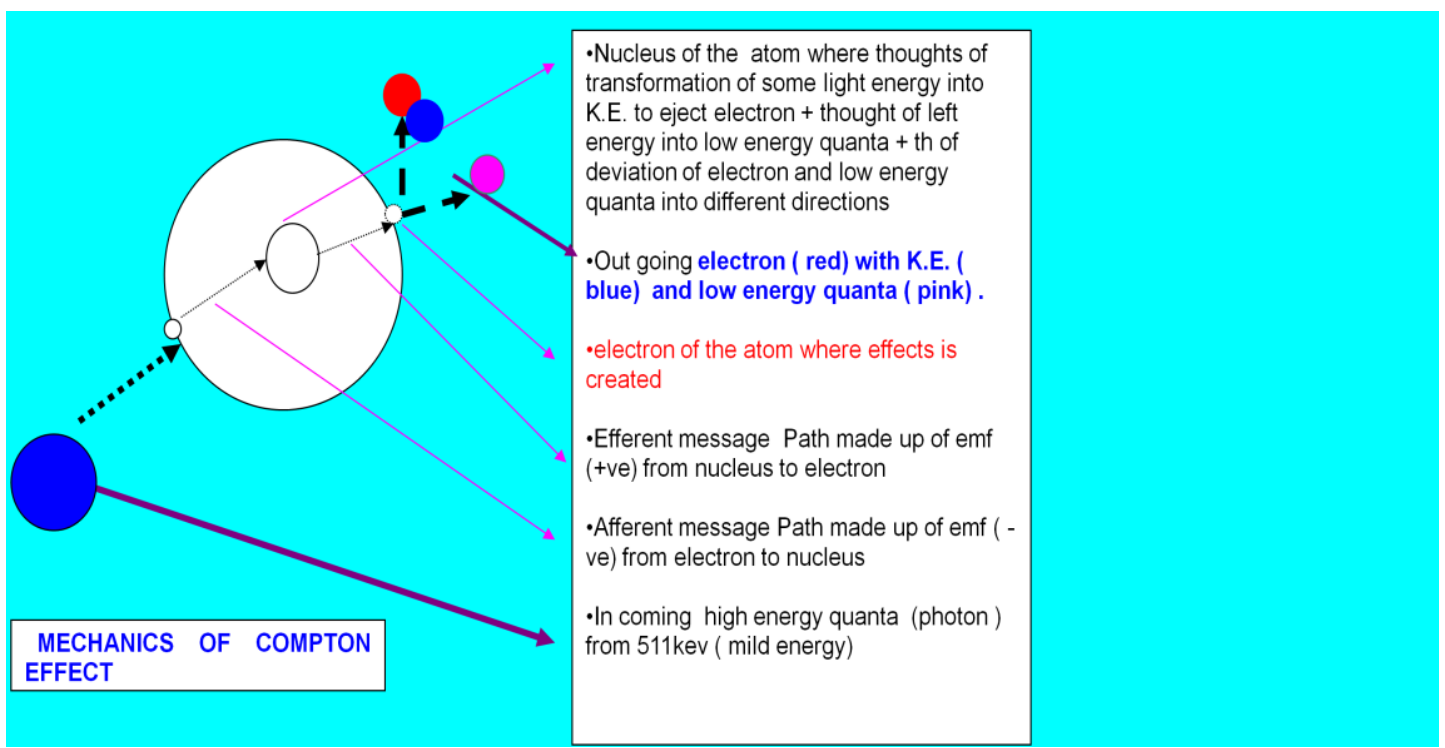

( figure 38 compton effect )

\section{PAIR PRODUCTION}

1. thought of transformation of high energy quanta into kinetic energy of electron and positron.

2. Thought of formation of electron and positron from nucleons.

Proton / neutron $\longrightarrow$ Quark $\longrightarrow$ energized gravitons + Binding energy

\section{C.C.P}

electrons + positrons

3. Thought of deviation of these electron and positron into different directions . 


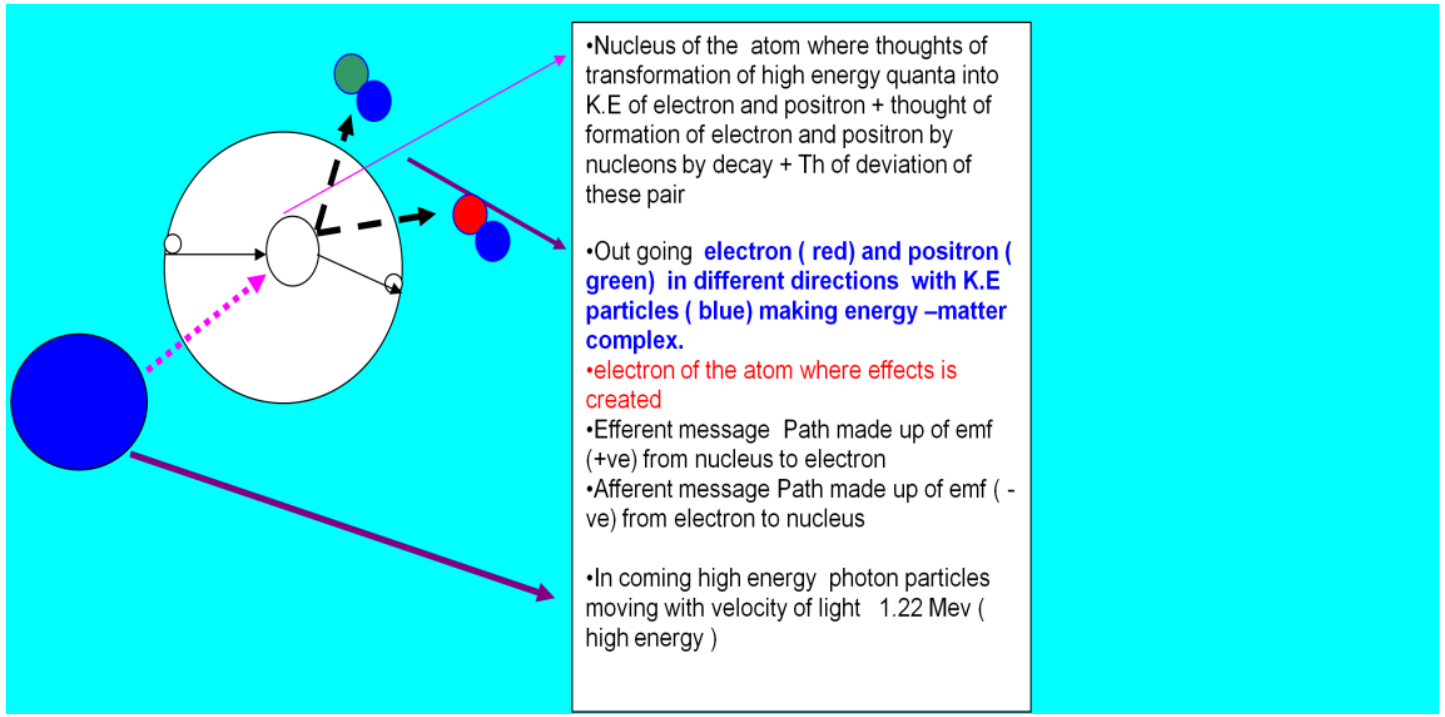

( figure 39 pair production )

\section{MECHANICS OF PAIR PRODUCTION figure 40, figure 41, figure 42}

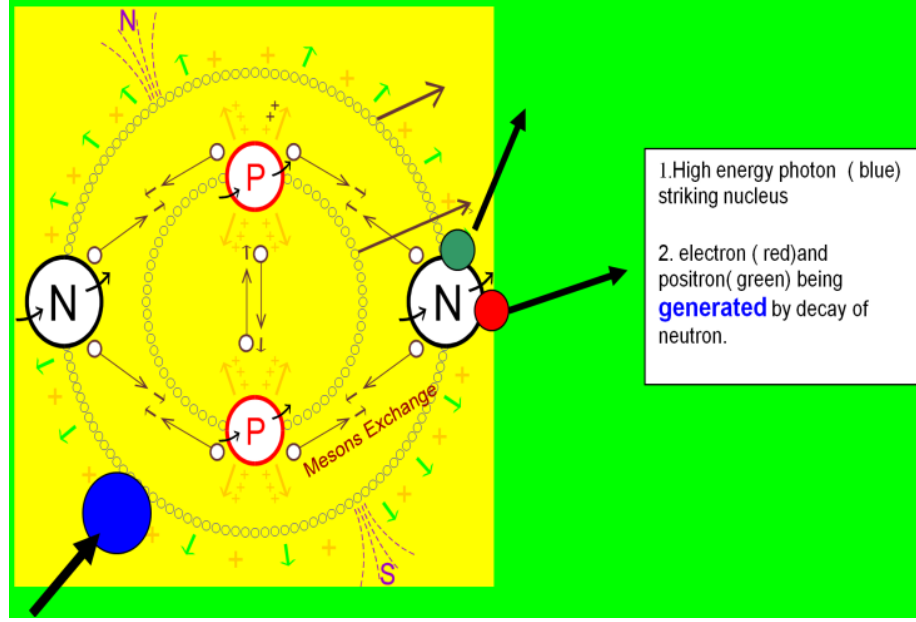

( figure 40 pair production )

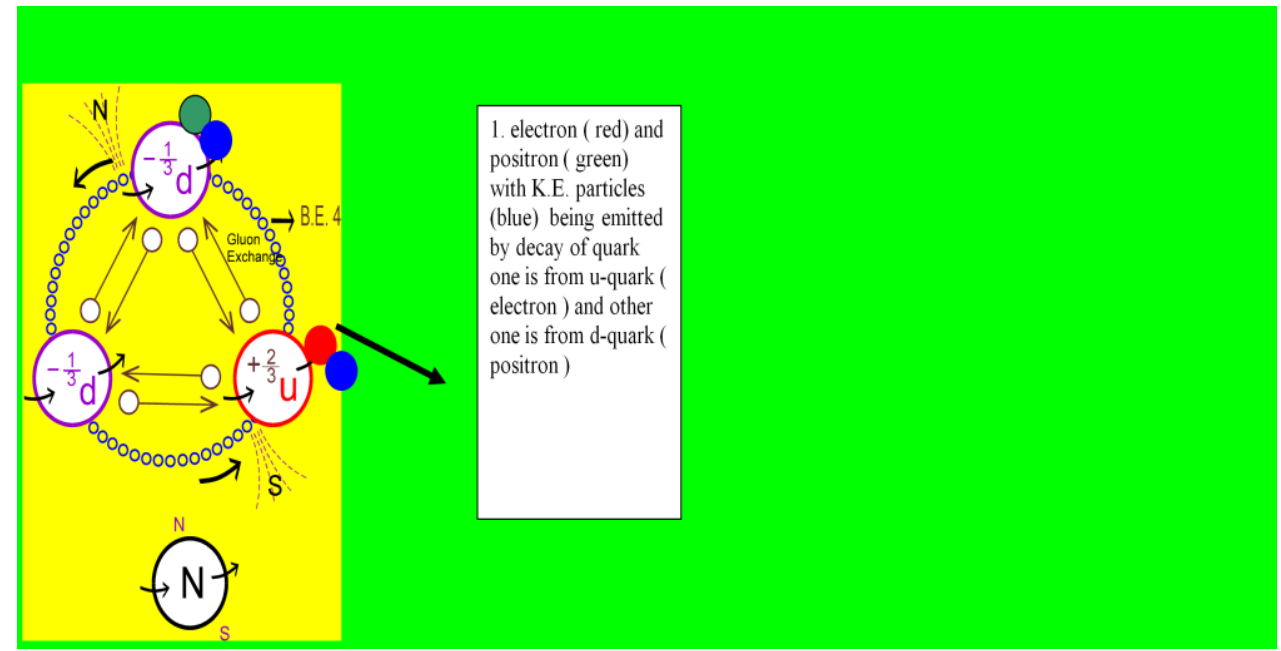

( figure 41 pair production ) 


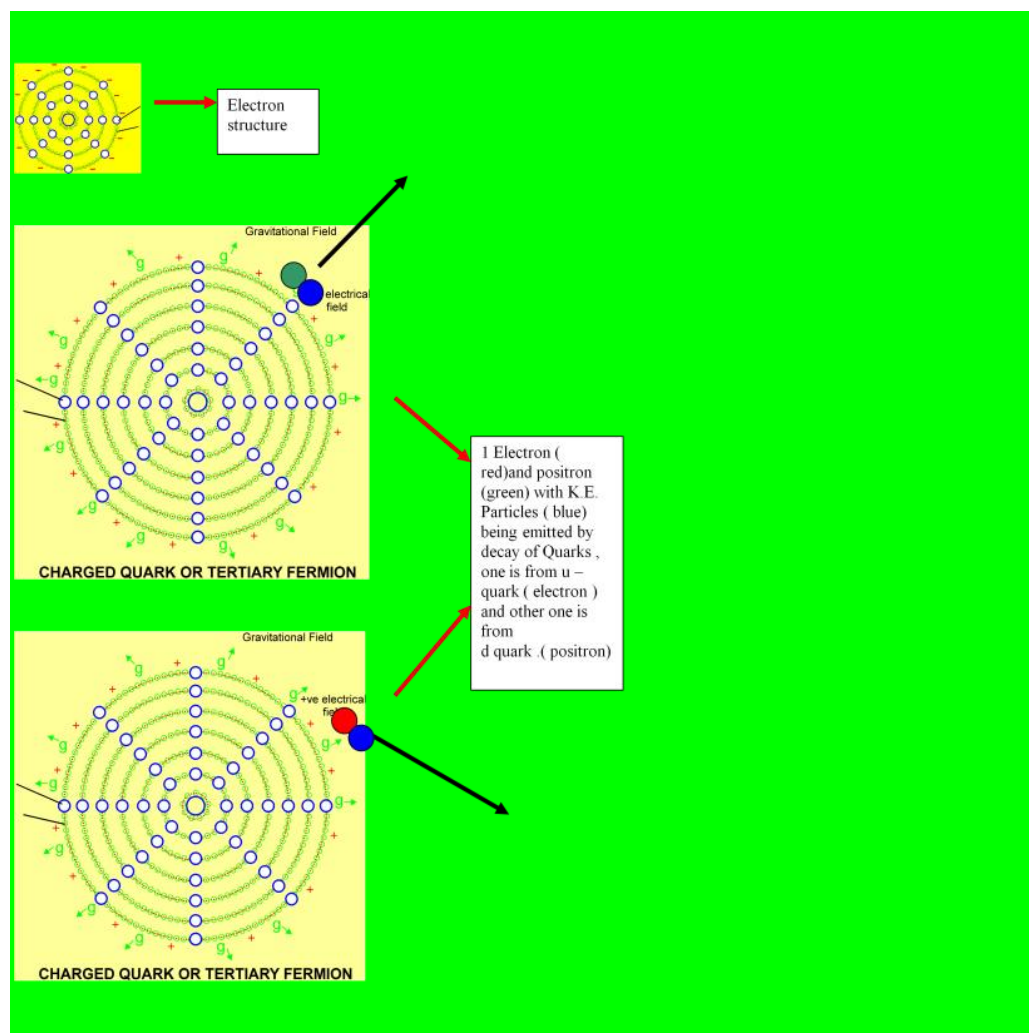

( figure 42 pair production )

Having triggered the thoughts by different stimuli, there is formation of messages in form of Code PCPs . These Code PCPs are carried by photons to different target B.B.B s. Having received the messages by different target B.B.Bs, they work accordingly and we observe effect in form of photo electric effect, Compton effect and pair production. All three effects are triggered by different stimuli ( conditioned stimulation of CCP ), having different thought generation and have different effects hence all three phenomena are different phenomena .

\section{Conclusions}

1. In pair production it is being believed that energy is transformed into matter ( electron and positron ). It is MYTH. Rather energy triggers nucleons to decay into electron and positron as destined by highest center of universe in pre creation era during programming of universe.

2. In photoelectric effect it is believed that electrons means current . It is myth. Electrons move from -ve to +ve side with drift velocity and they connect the two plates cathode and anode. While electrical energy or current moves from +ve to -ve as energy particle with velocity of light by phenomenon of conduction i.e. jumping on electrons.

3. In Compton effect the interaction of energy quanta and electron is not like playing billiard balls rather there is involvement of nucleus to decide the effect by triggering of thoughts . Unless thoughts are expressed in nucleus, effect cannot come. Same is true for photoelectric effect.

4. All the three effects profound that photon and atoms or matter are DIVINE.

5. It is believed that nucleons do not decay when they are bonded in nucleus . It is Myth . Pair production shows that nucleons do decay even if they are bonded in nucleus ( conditioned stimulation of CCP) .

6. If theory is not being explained by MIND, it is wrong theory. Hence theories given by Einstein for photo electric effect, Compton, are wrong theories as well as theory of pair production is also wrong.

7. $\mathrm{E}=\mathrm{mC} 2$ is myth. Hence calculations given in pair production are wrong.

\subsection{Abundance of Helium [18]}


3.

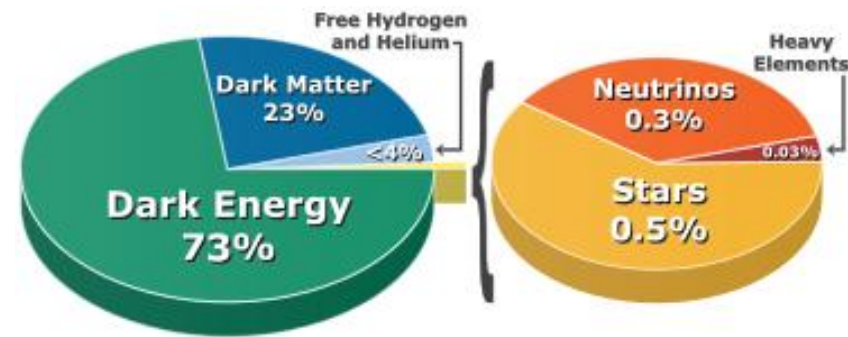

(Figure 43 -Chart shows the proportion of different components of the universe - about $95 \%$ is dark matter and dark energy.)

\section{Primordial gas clouds [18]}

In 2011 astronomers found what they believe to be pristine clouds of primordial gas, by analyzing absorption lines in the spectra of distant quasars. Before this discovery, all other astronomical objects have been observed to contain heavy elements that are formed in stars. These two clouds of gas contain no elements heavier than hydrogen and deuterium. ${ }^{[71][72]}$ Since the clouds of gas have no heavy elements, they likely formed in the first few minutes after the Big Bang, during Big Bang nucleosynthesis. Their composition matches the composition predicted from Big Bang nucleosynthesis. This provides direct evidence that there was a period in the history of the universe before the formation of the first stars, when most ordinary matter existed in the form of clouds of neutral hydrogen

\section{Abundance of primordial elements [18]}

Using the Big Bang model it is possible to calculate the concentration of helium-4, helium-3, deuterium, and lithium-7 in the universe as ratios to the amount of ordinary hydrogen. $\overline{\text { [22] }}$ The relative abundances depend on a single parameter, the ratio of photons to baryons. This value can be calculated independently from the detailed structure of $\mathrm{CMB}$ fluctuations. The ratios predicted (by mass, not by number) are about 0.25 for $4 \mathrm{He} / \mathrm{H}$, about $10^{-3}$ for $2 \mathrm{H} / \mathrm{H}$, about $10^{-4}$ for $3 \mathrm{He} / \mathrm{H}$ and about $10^{-9}$ for $7 \mathrm{Li} / \mathrm{H}$. ${ }^{22]}$ The measured abundances all agree at least roughly with those predicted from a single value of the baryon-to-photon ratio. The agreement is excellent for deuterium, close but formally discrepant for $4 \mathrm{He}$, and off by a factor of two $7 \mathrm{Li}$; in the latter two cases there are substantial systematic uncertainties. Nonetheless, the general consistency with abundances predicted by Big Bang nucleosynthesis is strong evidence for the Big Bang, as the theory is the only known explanation for the relative abundances of light elements, and it is virtually impossible to "tune" the Big Bang to produce much more or less than $20-30 \%$ helium. ${ }^{[68]}$ Indeed there is no obvious reason outside of the Big Bang that, for example, the young universe (i.e., before star formation, as determined by studying matter supposedly free of stellar nucleosynthesis products) should have more helium than deuterium or more deuterium than $3 \mathrm{He}$, and in constant ratios, too.

In quasars where apart from formation of hydrogen atoms from tachyons, nature also formed some other elements from created hydrogen by unconditioned working of thought expressions. If we see spectrum of quasars, we could see those elements which are being created there. Quasar redshifts are measured from the strong spectral lines that dominate their optical and ultraviolet spectra. These lines are brighter than the continuous spectrum, so they are called 'emission' lines. They have widths of several percent of the speed of light. These widths are due to Doppler shifts caused by the high speeds of the gas emitting the lines. Fast motions strongly indicate a large mass. Emission lines of hydrogen (mainly of the Lyman series and Balmer series), helium, carbon, magnesium, iron and oxygen are the brightest lines. The atoms emitting these lines range from neutral to highly ionized, i.e., many of the electrons are stripped off the ion, leaving it highly charged. This wide range of ionization shows that the gas is highly irradiated by the quasar, not merely hot, and not by stars, which cannot produce such a wide range of ionization. Iron quasars show strong emission lines resulting from low ionization iron (FeII), such as IRAS 18508-7815. [2]

In nuclear chemistry terms, deuterium nuclei, ${ }^{2} \mathrm{H}$, are very reactive. For several minutes the deuterium nuclei, ${ }^{2} \mathrm{H}$, reacted by a variety of nuclear reactions to give a mixture of isotopes: ${ }^{3} \mathrm{He},{ }^{4} \mathrm{He},{ }^{7} \mathrm{Li}$, along with the PRIMORDIAL ${ }^{1} \mathrm{H}$ and ${ }^{2} \mathrm{H}$. A graph, from here, shows the $(\log )$ time EVOLUTION of the abundances of the light ELEMENTS:[19] 


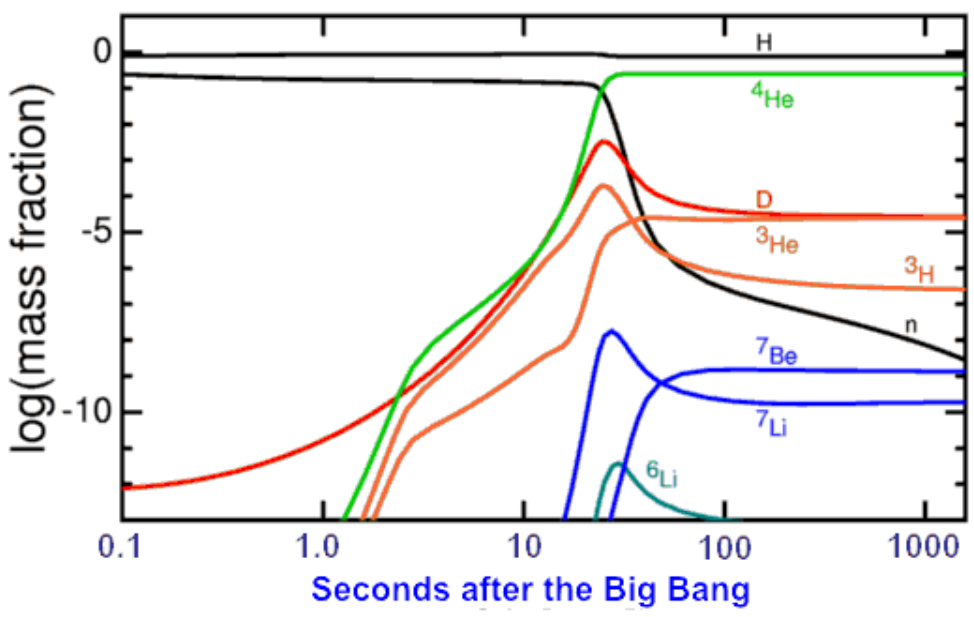

( Figure 44 Ratio of H, H2, He3, He4, Li7 in early universe [19] )

The ratios of ${ }^{1} \mathrm{H},{ }^{2} \mathrm{H},{ }^{3} \mathrm{He},{ }^{4} \mathrm{He}$ and ${ }^{7} \mathrm{Li}$ in the early universe can be measured by astronomers - with considerable - and the numbers obtained CONSTRAIN the mass, temperature and density conditions at this epoch.[19]

It is to be noted that (These two clouds of gas contain no elements heavier than hydrogen and deuterium. [71]172] Since the clouds of gas have no heavy elements, they likely formed in the first few minutes after the Big Bang, during Big Bang nucleosynthesis.) along with hydrogen, helium was not created in quasar to begin with . Before pure hydrogen cloud or nebula gets into proto stars formation, helium too comes into play at the tune of 20- $30 \%$. It means formation of helium is being taking place while hydrogen clouds transforming into proto stars . This could only be possible by unconditioned working of nature during formation of proto stars.

\subsection{Confirmation of Hoyle Narlikar Universe}

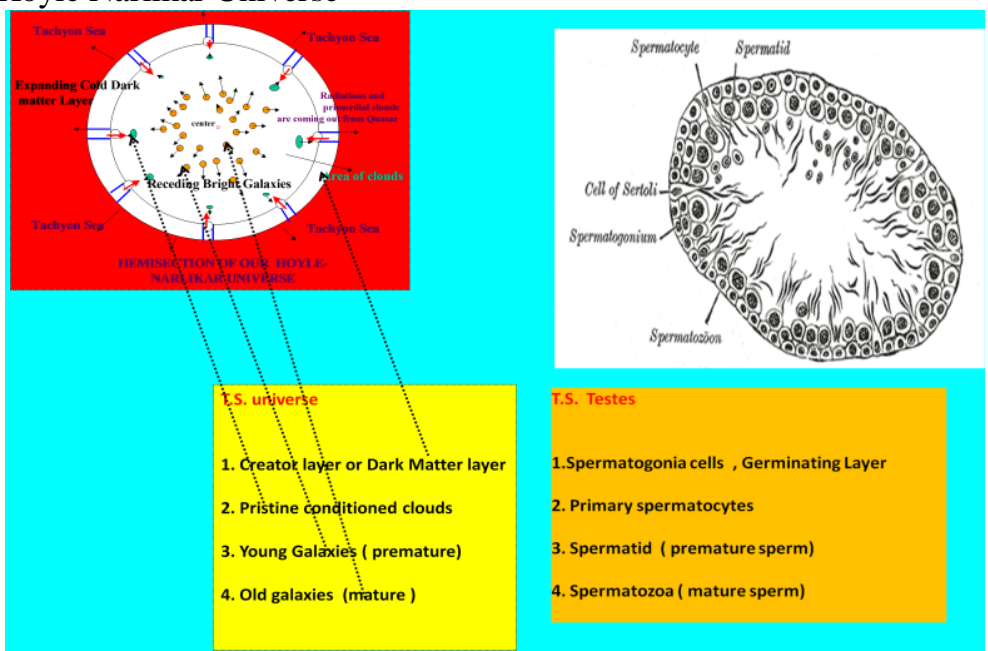

( Figure 45 T.S. Of Universe showing evolution of universe and T.S. of testes of man showing evolution or maturation of sperm)

Understanding the universe model . through Structural and Functional Analogies

Analogies if become available are helpful in understanding a physical system. In general, analogy means some kind of mental construct, which is useful in understanding the "unknown" in terms of the "known". Literally, the word "analogy" means similarity or parallelism. This similarity could be just of a concept or a sequence of concepts or a description of a model or the phenomenon as a whole. Further, these analogies could be well within the same discipline or in different disciplines of knowledge. Here are T.S. of Universe and T.S. of human testes . If you consider anatomy of both, both have some kind of similarities . These similarities are 1. There is Germinating layer which corresponds with creator layer of universe model. 2. Pristine conditioned clouds corresponds primary spermatocytes ( early premature sperms ) of testes section . 3. Spermatids ( young premature sperms ) corresponds young galaxies . 4. And spermatozoa corresponds mature or old galaxies of universe model . T.S of testes shows maturing stages of sperm towards center of testes . Similarly T.S. of universe shows maturing of galaxies towards center of the universe. Both the processes are triggered and controlled by Mind. It does show that model made by participatory science of universe origin by continuous 
creation is right model . It does also show that Almight B.B.B has disguised map of universe model in human testes. So that astrophysicists could not search it. Hence they are unknown about realities of the universe . But my conscious could search it and it drew the map of the universe in 1987 ( figure 46). After this I started testing my model and on 14 Feb 1090, I made a diagram of Quasar ( figure 47) where I drew creation of hydrogen . This is one of the secrete of the research .

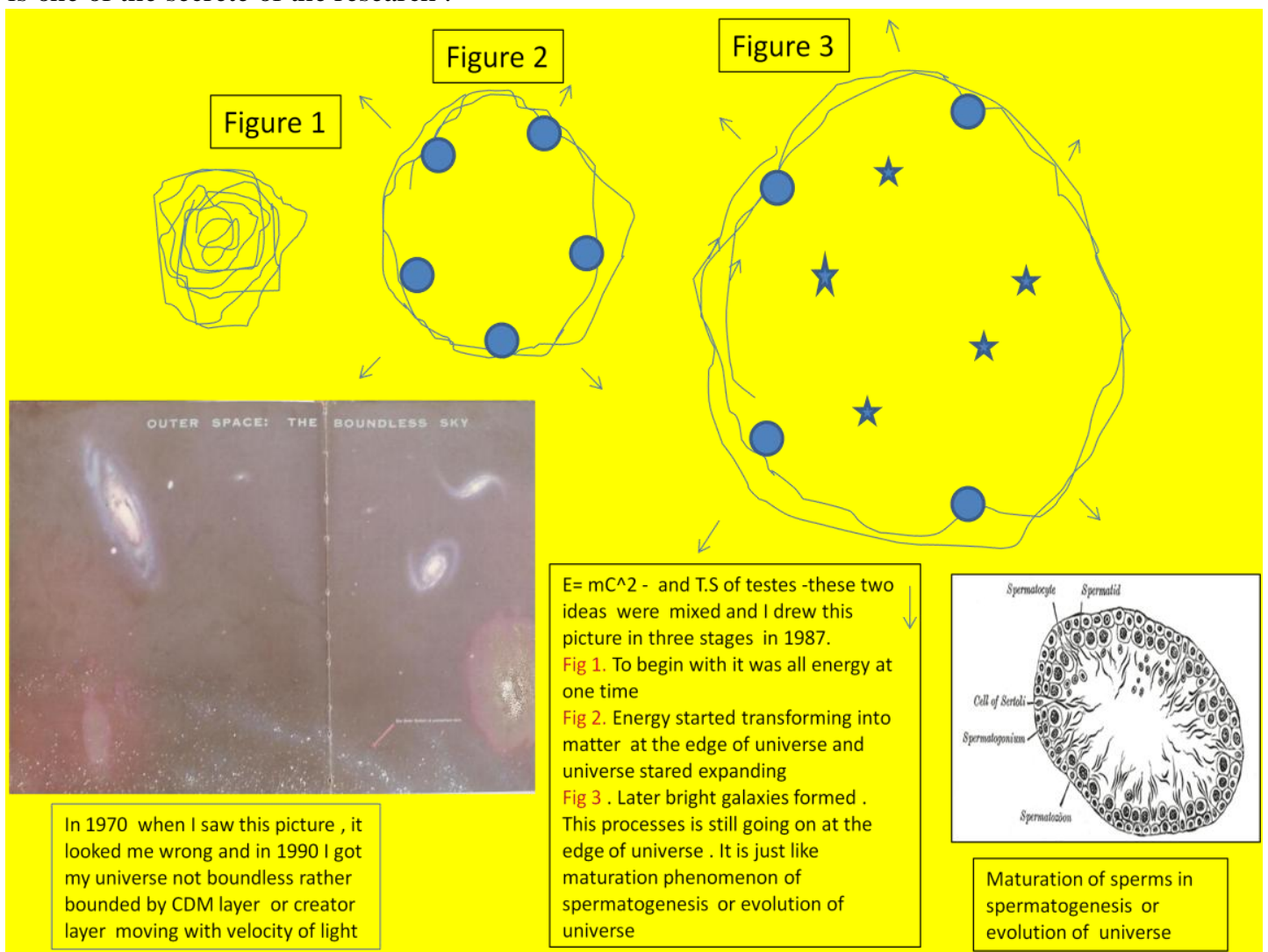

( Figure 46 - My ideas taking shape since 1970 to 1987 )

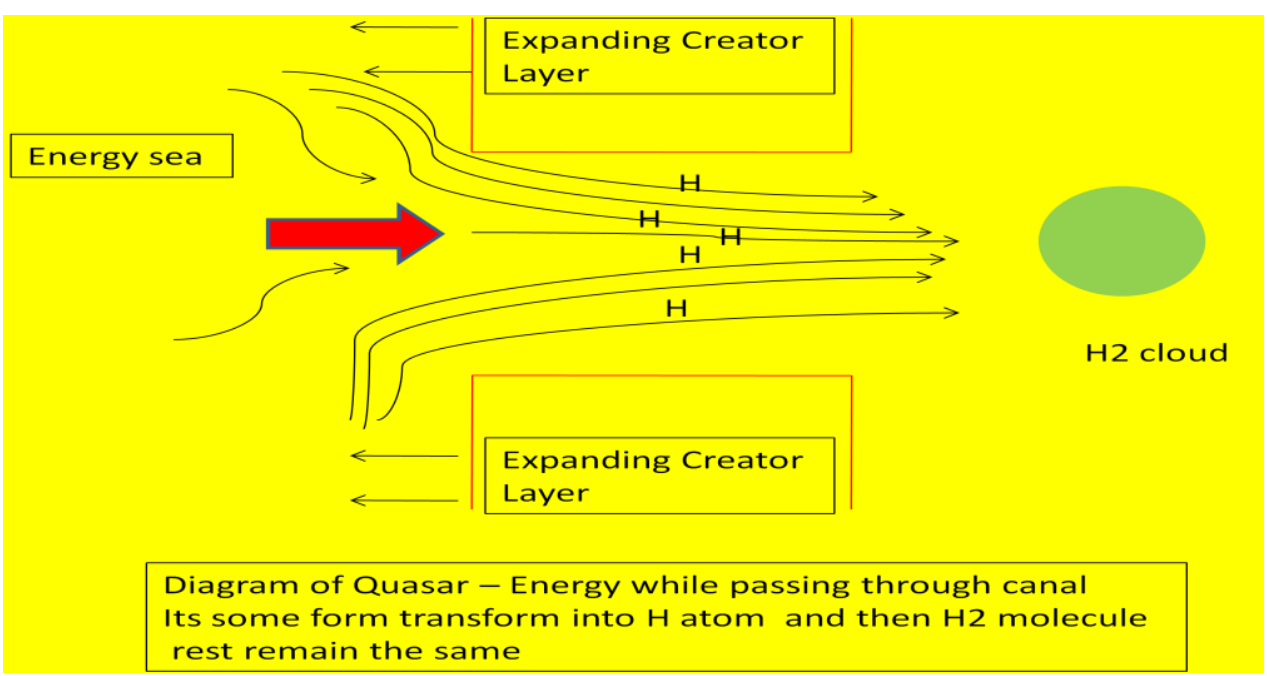

( Figure 47- creation of Hydrogen in quasar from 1987 to 1990 ) 


\subsection{Study of universe origin at two different Hubble constant}
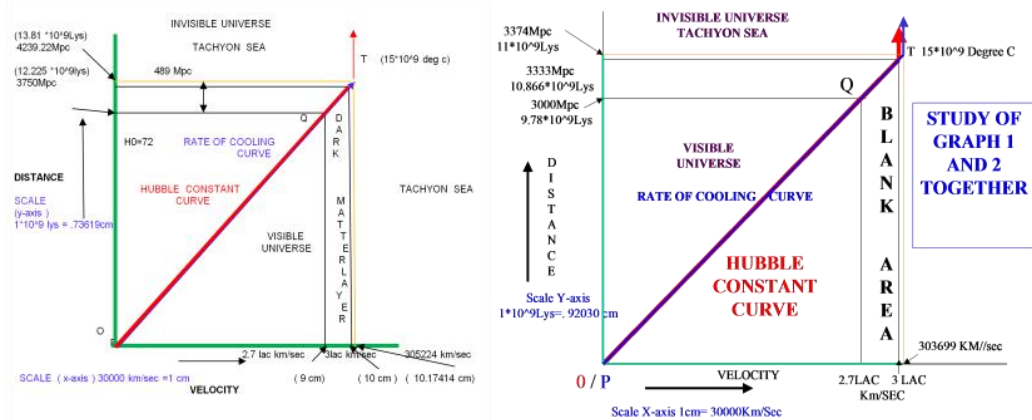

( Figure 46 - Study of universe origin at two different Hubble constant $72 \mathrm{~km} / \mathrm{sec} / \mathrm{Mpc}$ and $90 \mathrm{~km} / \mathrm{sec} / \mathrm{Mpc}$ )

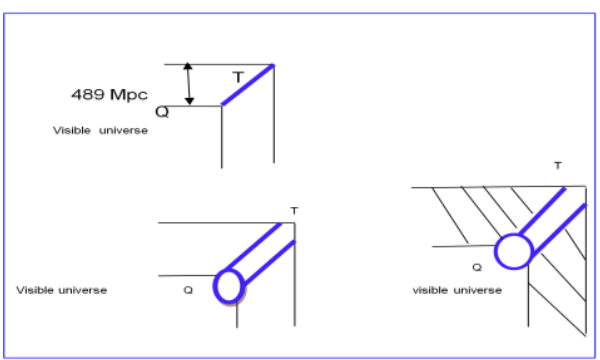

DIAGRAMS OF BLANK AREA HO $=72 \mathrm{~km} / \mathrm{sec} / \mathrm{MPC}$

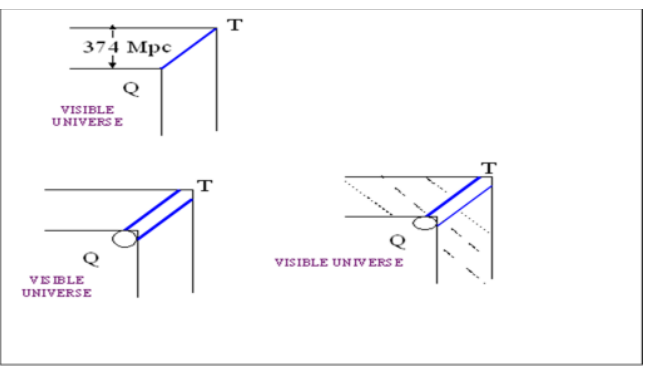

DIAGRAMS OF BLANK AREA HO $=90 \mathrm{~km} / \mathrm{sec} / \mathrm{Mpe}$

( Figure 47 Line diagram of CDM layer at two different Hubble constant )

I have done two studies at Hubble constant $=90 \mathrm{~km} / \mathrm{sec} / \mathrm{Mpc}$ and Hubble constant $=72 \mathrm{~km} / \mathrm{sec} / \mathrm{Mpc}($ Figure 46 and Figure 47 )and I found no change in the model of the universe and origin of the universe except that universe was small at $\mathrm{H} 0=90 \mathrm{~km} / \mathrm{sec} / \mathrm{Mpc}$ and it is big at $\mathrm{H} 0=72 \mathrm{~km} / \mathrm{sec} / \mathrm{Mpc}$. In expanding universe Ho is retarding. Hence no matter at what Ho the study is performed, the model and origin of the universe remain the same except that we see different stages of the universe with their changing size . One thing more we observe that thickness of CDM layer increases with decrease in Hubble constant. It means rate of expansion of galaxies is different in different regions of the universe as retardation of galaxies is not uniform. It changes with the time as Hubble constant is retarding . H0 does not show rate of expansion of universe as universe is expanding with velocity of light. Hence olber's paradox is there. Had it been expanding less than velocity of light, there would be no olber's paradox. H0 shows expansion rate of galaxies which are also retarding . Hence Ho is retarding . It is all due to Cold dark matter layer which is forming boundary of universe and receding with velocity of light . Its thickness is increasing with time and that changes rate of expansion of galaxies and value of Hubble constant. Different values of H0 show different times or stages of the expanding universe. This shows divine and changing nature of our universe.

\subsection{Atomicgenetics and sound phenomenon}

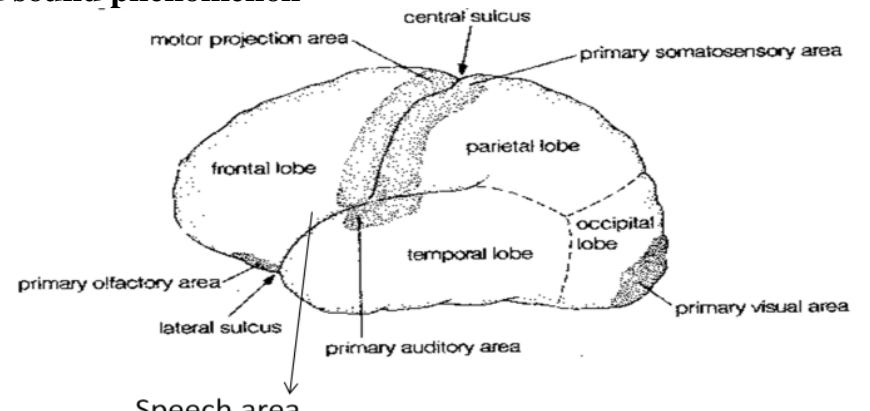


( Figure 48 - surface of brain and speech area )

PATH FROM SPEECH AREA TO VOCAL CORDS

Speech area in frontal lobe ( figure 48) --- to RS --- arousal phenomenon ---motor cortex nuclei ( vocal cord, muscle of tongue, Lips ) --- cortico- pontine tract $\left(7^{\text {th }}\right.$ cranial nerve for facial muscle $)$ and cortico medulary tact $\left(10^{\text {th }}\right.$ cranial nerve and $12^{\text {th }}$ cranial nerve - for tongue muscles $)$. From $10^{\text {th }}$ cranial nerve nucleus - nucleus ambiguous $-10^{\text {th }}$ nerve - recurrent laryngeal nerve ---- muscles of larynx ( vocal cords vibration)--sound effect

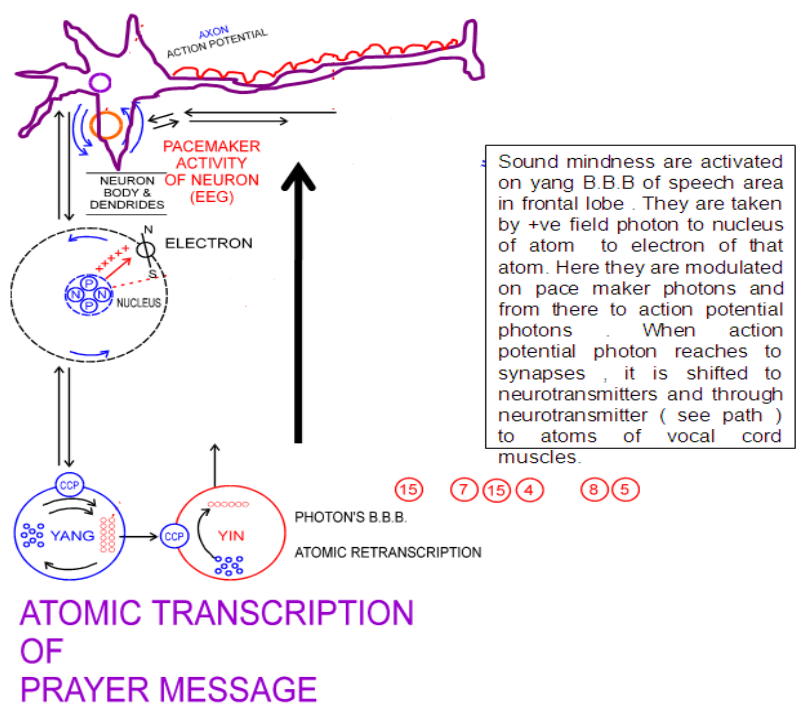

( Figure 49-Generation of sound mindness on Yang B.B.B and carried by photon )

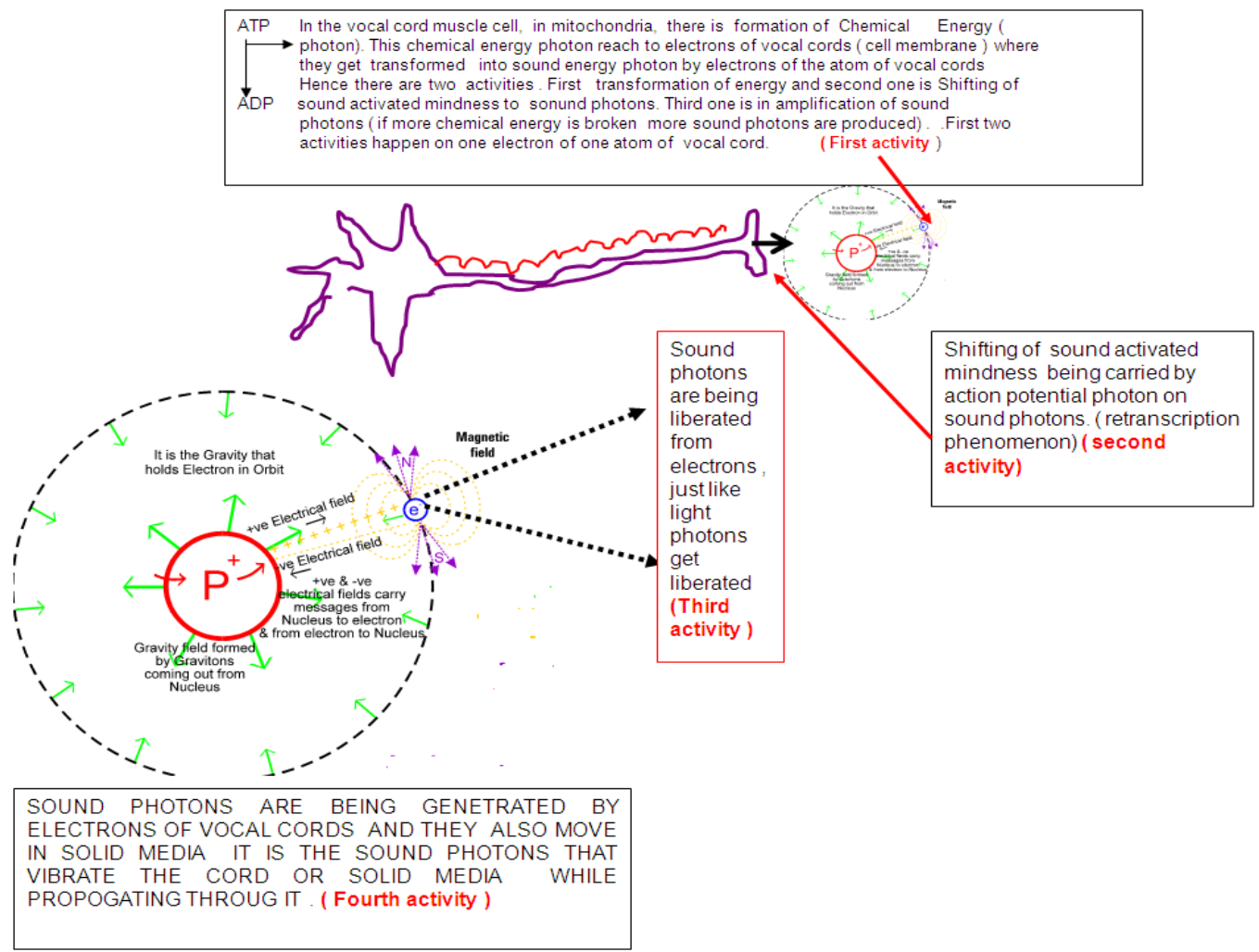

( Figure 50 - Photon carrying sound mindness from speech area to vocal cord ) 

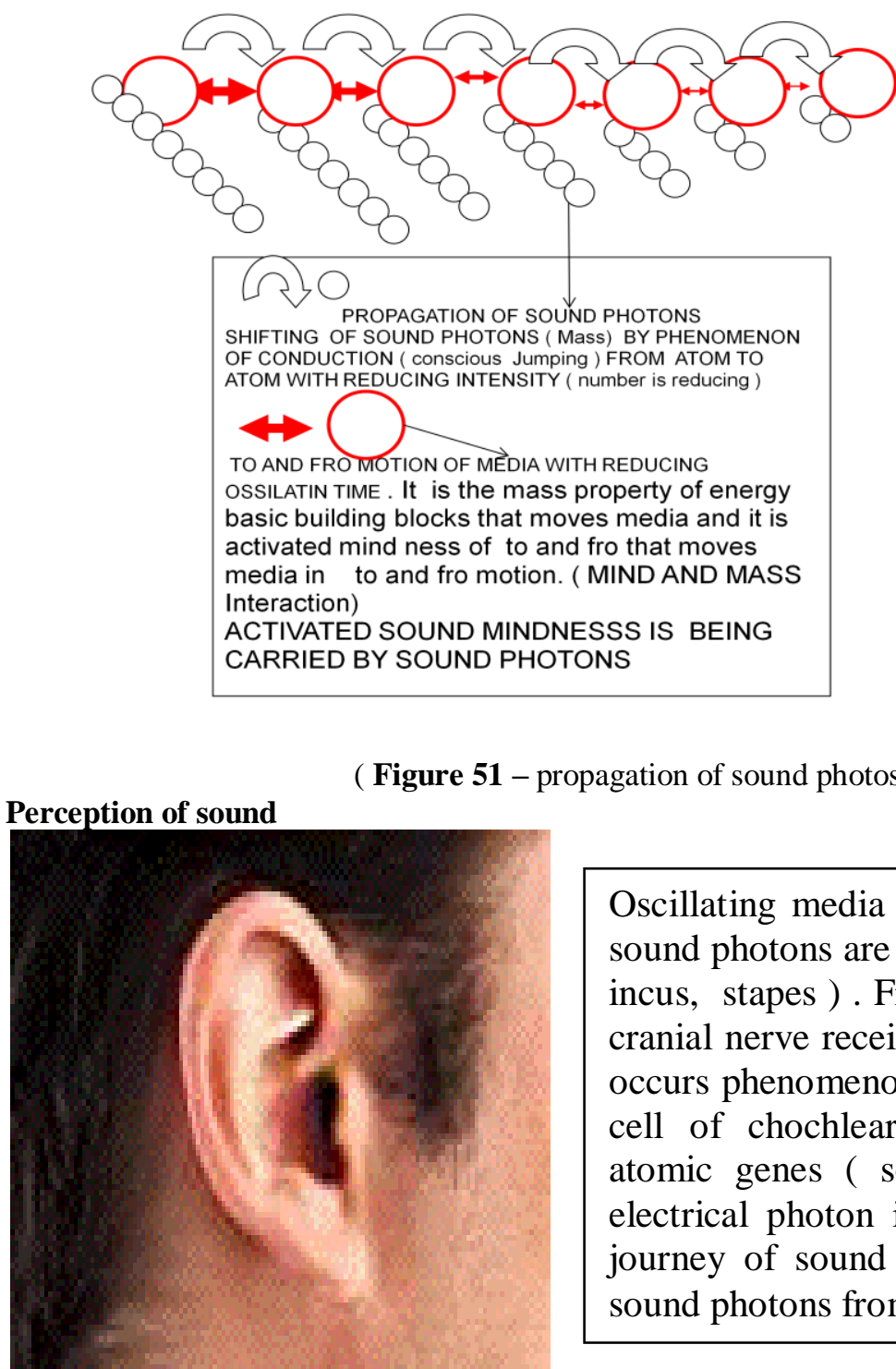

( Figure 51 - propagation of sound photos in media -air )

( Figure 52 - sound photons being received by ear )

ACOUSTIC NERVE Understanding the Ear

- Outer Ear: The outside of the ear, plus the ear canal up to the eardrum or tympanic membrane.

- Middle Ear: The cavity behind the tympanic membrane which houses three small bones that help conduct sound waves to the inner ear.

- Inner Ear: The cavity behind the middle ear houses a series of channels known as the labyrinth, and a snailshaped structure called the cochlea. The labyrinth is integral to the human balance system. The cochlea contains the thousands of nerves responsible for sensing sound and transmitting it to the brain for processing.

- Auditory Nerve: The eighth cranial nerve, which is the pathway of sound from the ear to the brain. 


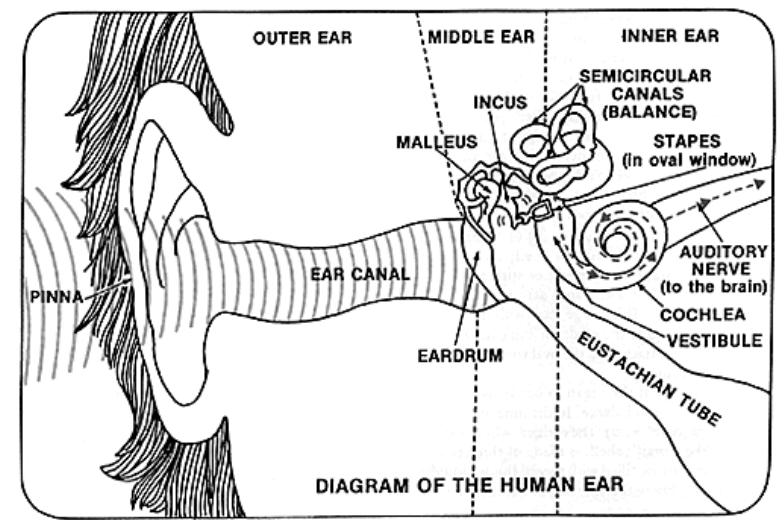

( Figure 53- journey of sound photons and sound mindness in side ear )

The vestibulocochlear nerve consists mostly of bipolar neurons and splits into two large divisions: the cochlear nerve and the vestibular nerve.

The cochlear nerve travels away from the cochlea of the inner ear where it starts as the spiral ganglia. Processes from the organ of Corti conduct afferent transmission to the spiral ganglia. It is the inner hair cells of the organ of Corti that are responsible for activation of afferent receptors in response to pressure waves reaching the basilar membrane through the transduction of sound. The exact mechanism by which sound is transmitted by the neurons of the cochlear nerve is uncertain; the two competing theories are place theory and temporal theory.

The vestibular nerve travels from the vestibular system of the inner ear. The vestibular ganglion houses the cell bodies of the bipolar neurons and extends processes to five sensory organs. Three of these are the cristae located in the ampullae of the semicircular canals. Hair cells of the cristae activate afferent receptors in response to rotational acceleration. The other two sensory organs supplied by the vestibular neurons are the maculae of the saccule and utricle. Hair cells of the maculae activate afferent receptors in response to linear acceleration.

Sound waves enter the outer ear (pinna) and travel through a narrow tube (ear canal) that leads inside the ear to the eardrum (tympanic membrane). The eardrum vibrates from the incoming sound waves and transmits these vibrations through three tiny bones called the ossicles (the malleus, incus, and stapes) in the middle ear. They amplify the sound and send it through the entrance to the inner ear (oval window) and into the fluid-filled hearing organ (cochlea).

The vibrations create ripples in the fluid that bend projections from tiny hair cells in the cochlea, causing electrical impulses that the auditory nerve, or eighth cranial nerve, sends to the brain.

The brain translates these impulses into what we experience as sound.

\section{PATH OF EIGHTH CRANIAL NERVE ( FROM CHOCHLEA TO HEARING CENTER IN TEMPORAL LOBE ( PERCEPTION PHASE -1)}

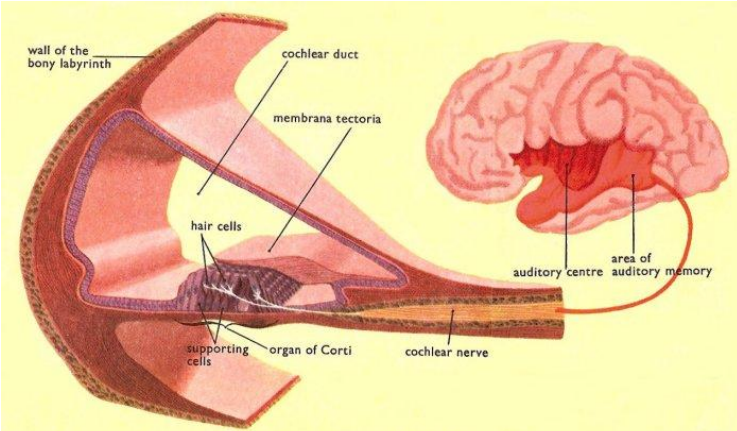

( Figure 54 - Journey of sound mindness fron organ of corti to temporal lobe by action potential )

The organ of Corti (or spiral organ) is the organ in the inner ear found only in mammals that contains auditory sensory cells, or "hair cells."

The organ was named after the Italian anatomist Marquis Alfonso Giacomo Gaspare Corti (18221876), who conducted microscopic research of the mammalian auditory system.

[edit] Structure and function

See also: Stereocilia (inner ear)

The organ of Corti has highly specialized structures that respond to fluid-borne vibrations in the cochlea with a shearing vector in the hairs of some cochlear hair cells. It contains between 15,000-20,000 
auditory nerve receptors. Each receptor has its own hair cell. The shear on the hairs opens non-selective transduction ion channels that are permeable to potassium and calcium, leading to hair cell plasma membrane depolarization, activation of voltage-dependent calcium channels at the synaptic basolateral pole of the cells which triggers vesicle exocytosis and liberation of glutamate neurotransmitter to the synaptic cleft and electrical signaling to the auditory cortex via spiral ganglion neurons. The pinna and middle ear act as mechanical transformers and amplifiers, so that by the time sound waves reach the organ of Corti, their pressure amplitude is 22 times that of the air impinging on the pinna. The organ of Corti can be damaged by excessive sound levels, leading to noise-induced health effects. The organ of Corti is the structure that transduces pressure waves to action potentials. The organ of Corti sits inside the cochlear duct, between the scala vestibuli and the scala tympani. The basilar membrane on the scala tympani presses against the hair cells of the organ as perilymphatic pressure waves pass.

REALISATION PHASE ( 2 PHASE ) ) ( FROM TEMPORAL LOBE TO RAS ) Reticular Activating System ( CONTROL LOOP )

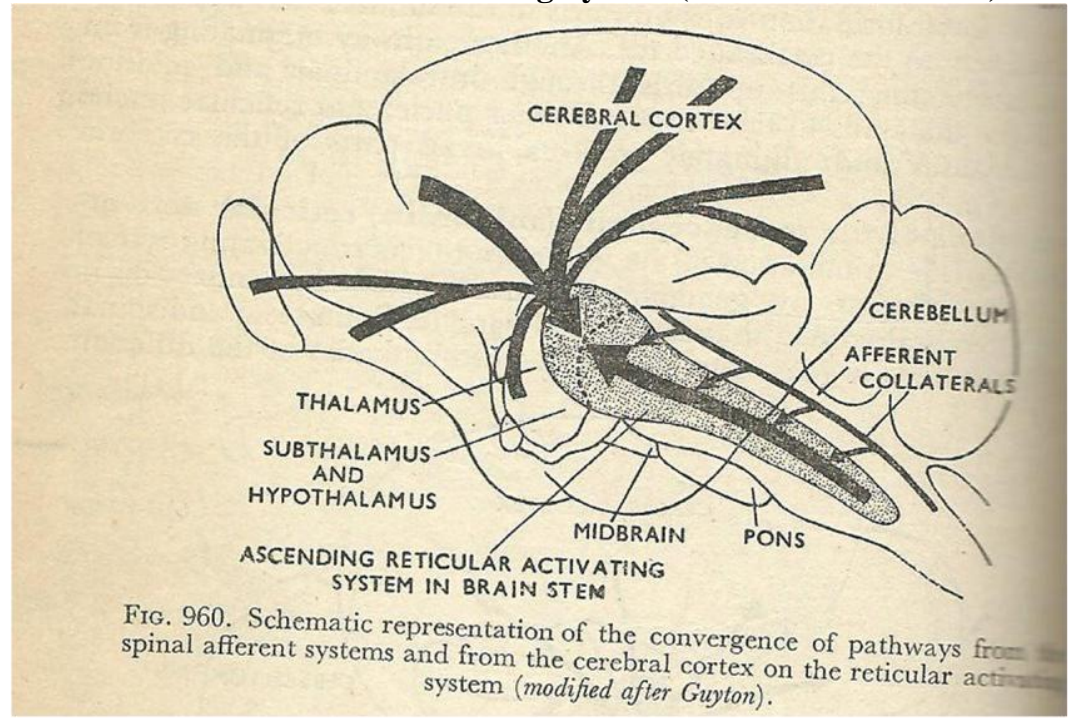

(Schematic representation of conversion of pathways from the spinal afferent system and from cerebral cortex on the reticular activating system (RAS different messages are realized here )

( Figure 55 - journey of sound mindness from temporal lobe to RS ) propagating action potentials

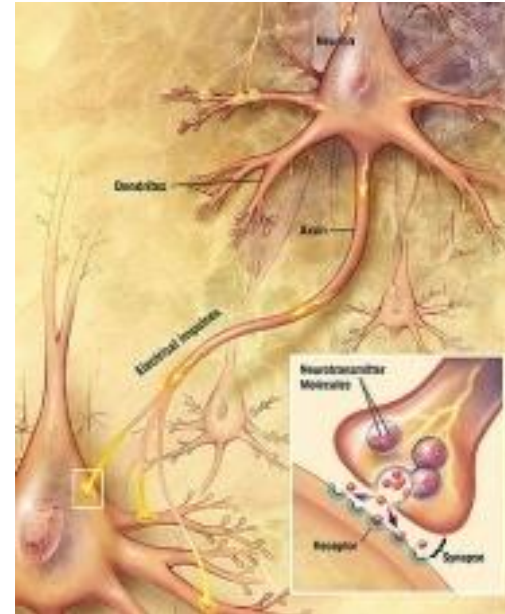

(Figure 56 -A signal propagating down an axon to the cell body and dendrites of the next cell) 


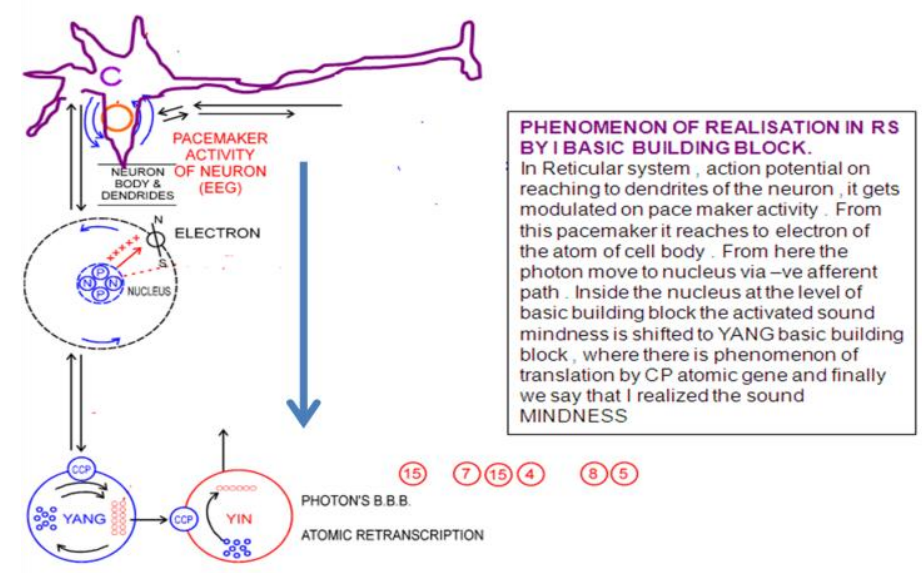

( Figure 57 - journey of sound mindness inside RS where it reaches final destination on Yang B.B.B where I realizes sound effect of O GOD HELP ME )

\section{JOURNEY OF ACTIVATED SOUND MINDNESS IS FROM YANG B.B.B ( SPEECH AREA)OF PRODUCER TO YANG B.B.B ( RETICULAR SYSTEM) OF RECIEVER}

2.10 Atomicgenetics and burning property of matter ( stone age phenomenon )

A match is typically a wooden stick (usually sold in match boxes) or stiff paper stick (usually sold in matchbooks) coated at one end (the match head) with a material often containing the element phosphorus, which will ignite from the heat of friction if rubbed ("struck") against a suitable surface IT IS WRONG STATEMENT

Man used to learn how to ignite matter due to friction even since stone age. There are many matter that get ignited due to friction but the mostly used matter or element is phosphorus or red phosphorus used in match box. When it is rubbed against friction surface it gets ignited. It is chemical property of the matter because there is chemical reaction involved in the process. But the question is how ignition is triggered ?

1. WITH TRANSFORMATION OF MATTER

2. WITHOUT TRANSFORMATION OF MATTER

To understand it we have to see Figure- 2 and Figure -3. . There are two types of thought stimulation . One is CONDITIONED THOUGHT STIMULATION and other one is UNCONDITIONED THOUGHT STIMULATION. During ignition phenomenon, conditioned stimulation of thought is there . It means that stimulus is from out side and it is in from of friction or Kinetic energy particles or mechanical energy photons. 


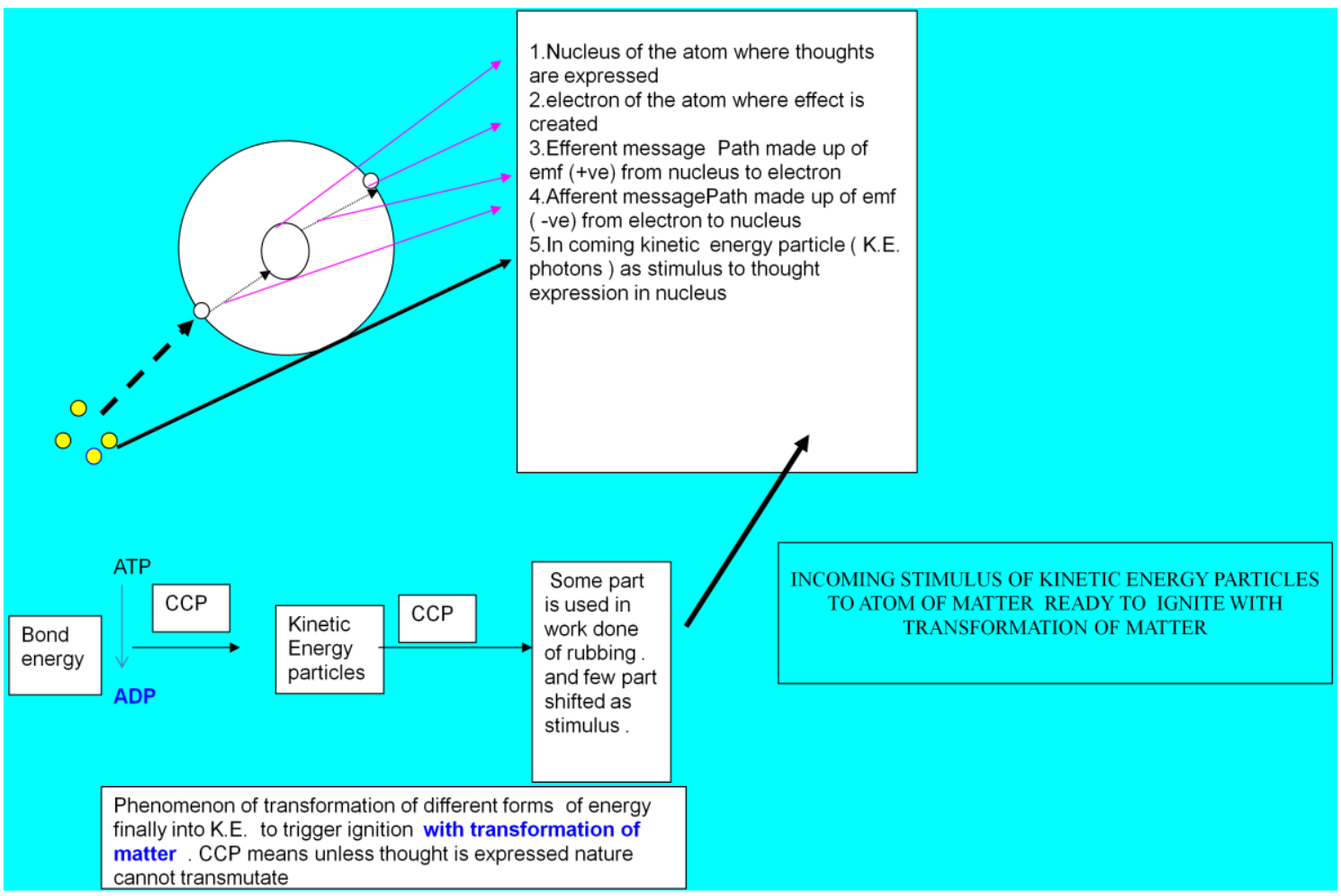

\section{DIFFERENT TYPES OF THOUGHT EXPRESSIONS TO IGNITE THE MATTER WHEN THE} KINETIC ENERGY STIMULUS HAS COME

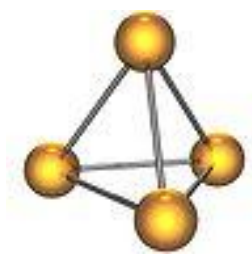

$\mathrm{P}_{4}$ molecule

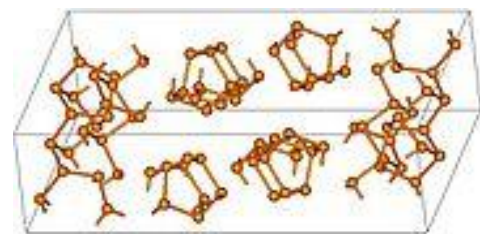

$\square$ Crystal structure of red phosphorus

Phosphorus has several forms (allotropes) which have strikingly different properties. ${ }^{[9]}$ The two most common allotropes are white phosphorus and red phosphorus

Types of thought expressions

1. Thought of transformation of phosphorus with oxygen into phosphorus oxide.

2. Thought of transformation of chemical bond energy into heat energy, light and sound energy.

With the result there are formation of messages in form of Code PCPs ( messenger atomic genes ) which are carried by efferent path made up of +ve emf from nucleus to electron. The messages get spread to all matter i.e. to all the atoms of phosphorus and oxygen in contact and chemical energy particles ( bond energy ) . Having received the messages they work accordingly and finally we observe the phenomenon as effect where heat and light and sound are being liberated or generated with formation of oxide of the matter. During transformation of matter into oxide, its physical, chemical, structural and spectral properties are suppressed ( of both phosphorus and oxygen molecules) while the new properties of oxide of matter ( structural , physical chemical and spectral ) are now dominant.

\section{Equation could be written as}




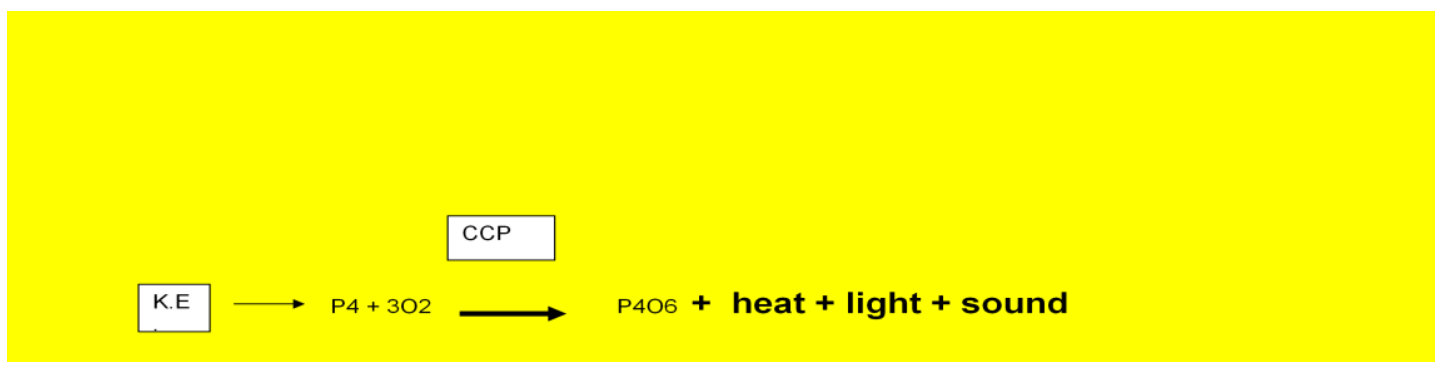

( CCP means Fig 2 and Fig 3 i.e. unless thought is expressed by conditioned stimulation, chemical reaction of ignition could not trigger or proceed to right side. )

During phenomenon of transmutation or chemical reaction ,there are many thoughts which are suppressed ( of left side of equation ) and there are many thoughts which are triggered ( of right side of equation) during the entire phenomenon of ignition but to understand the concept I have depicted two thoughts of activation ( of left side of equation ) only.

[edit] Oxides and oxyacids - Phosphorus(III) oxide, $\mathrm{P}_{4} \mathrm{O}_{6}$ (also called tetraphosphorus hexoxide) and phosphorus(IV) oxide, $\mathrm{P}_{4} \mathrm{O}_{10}$ (or tetraphosphorus decoxide) are acid anhydrides of phosphorus oxyacids and hence readily react with water. $\mathrm{P}_{4} \mathrm{O}_{10}$ is a particularly good dehydrating agent that can even remove water from nitric acid, $\mathrm{HNO}_{3}$. The structure of $\mathrm{P}_{4} \mathrm{O}_{6}$ is like that of $\mathrm{P}_{4}$ with an oxygen atom inserted between each of the $\mathrm{P}-\mathrm{P}$ bonds. The structure of $\mathrm{P}_{4} \mathrm{O}_{10}$ is like that of $\mathrm{P}_{4} \mathrm{O}_{6}$ with the addition of one oxygen bond to each phosphorus atom via a double bond and protruding away from the tetrahedral structure.

\section{WHAT HAPPENS WHEN --}

When match sticks or match box are drenched in water, then on striking the match stick, it does not ignite or it ignites with difficulty Why?. Because water forms a film over the match stick or on match box. It prevent stimulus to go and trigger phenomenon of ignition. The entire stimulus ( kinetic energy ) is being absorbed by water. Till water molecules are there, either ignition would not be triggered or if stimulus is so strong that it could pass the barrier to trigger ignition formed by water molecules.
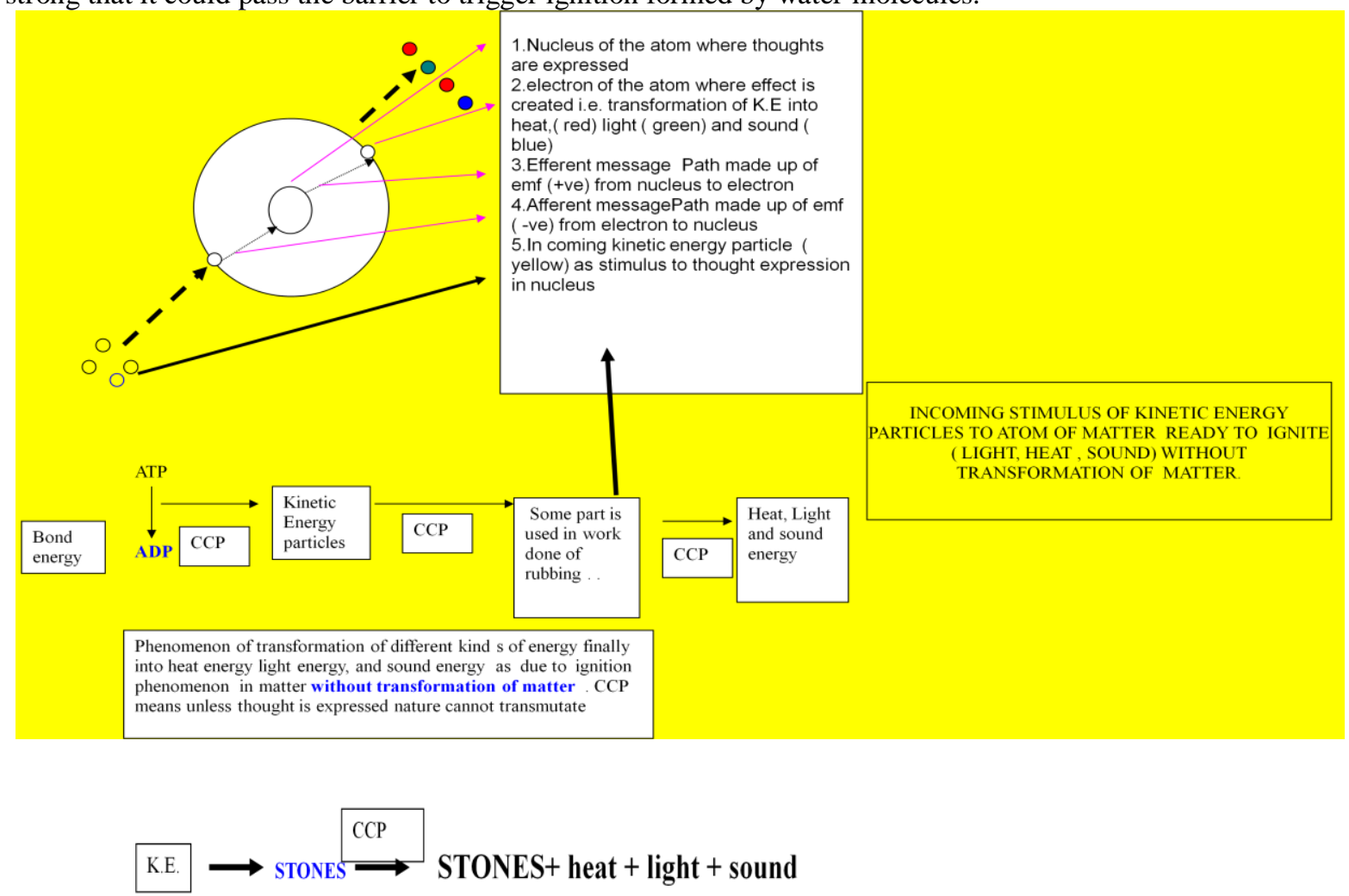

CATCHING OF FIRE IS DIFFERENT PHENOMENON ( THE STIMULUS IS HEAT ENERGY ) THAN IGNITION PHENOMENON ( STIMULUS IS KINETIC ENERGY PARTICLES) AND BOTH ARE TRIGGERED BY DIFFERENT THOUGHTS. 
CATCHING OF FIRE IS DIFFERENT PHENOMENON Paper is made from wood, so most of what is burning in paper is the same as what is burning in wood. That means mostly "cellulose", which is made up of the same elements as ordinary sugar(carbon, hydrogen, and oxygen) but in long polymerized chains.

You could see this phenomenon very well when paper catches fire when exposed directly to fire. But when you place water on it making bowel of paper and place it on fire, the paper does not catch fire till the temperature of paper reaches to that extent ( thresh hold level ) where it catches fire. Before that the entire heat is being absorbed by water placed inside it and it does not trigger catching of fire phenomenon of paper. The thoughts of paper of catching of fire phenomenon remains inactive till a fixed amount of heat stimulus is received by them to trigger the phenomenon.

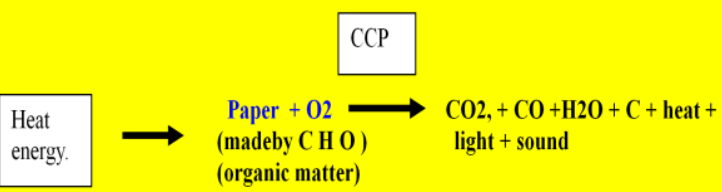

In match stick, ignition is done by phosphorus and catching of fire is done by wood stick. Similarly in stone age rubbing of stone triggers ignition and the dry grass catches the fire. Similarly in motor car, ignition is triggered by self ( battery, self starter, engine in motion, dynamo in motion, electricity generation , distributor, ) that ignite plugs ( spark - atoms transform electrical energy into spark i.e. heat, light and sound ) and the fire is caught by petrol

\section{Conclusion Of The Experiment.}

LAW OF IGNITION (TRIGGERING BY TRANSFORMATION OF MATTER );- The law states that during ignition by striking the matter ( PHOSPHORUS), the KINETIC energy triggers many thoughts of transmutation of matter into other matters ( oxidation phenomenon) as well as transformation of chemical energy into other form of energy ( heat, light and sound) which gives the effect of fire.

LAW OF IGNITION( TRIGGERING BY WITHOUT TRANSFORMATION OF MATTER. .) ;- The law states that during ignition by striking the matter ( metals, stones), the mechanical energy ( K.E.) triggers the thought of transformation of Kinetic energy into other form of energy ( heat, light and sound) with out the transformation of matter, which gives the effect of fire.

Both heat photons as well as phosphorus or metals and stones are divine units. They are not physical units . It means their working is triggered by atomic transcriptions or thought expressions.

\section{Discussion}

Modern scientists believe that heat energy (heat Photon) as well as phosphorus are physical units . Participatory science has proved them divine units and divinity is due to presence of atomic genes on basic building blocks ( B.B.Bs.), the fundamental particles and divine activity or fundamental activity of the nature is atomic transcription or thought expressions.

\section{Conclusions}

It is myth that we are stardust. The knowledge of structure of particles ( fermions and bosons ) is very important to understand annihilation and materialization of particles in particle physics . It is the mind that decides the particle interactions. The dark matter and dark energy knowledge is also very important to know particle's interaction in particles physics. Spin property of electrons and nucleus is important to have all interactions of the universe including physical, chemical and life sciences interactions as messages only could move when spin property of particles and nucleus exist. The gravity force could only work if there is spin property of these particles and nucleus. It is mind that moves more than velocity of light and it has been confirmed in prayer phenomenon. The theory of photoelectric effect, Compton effect and pair production are wrong as they are not explained by Mind . Abundance of Helium is due to unconditioned working of nature rather that Big Bang origin of the universe. New model of the universe has been confirmed by structural analogy of T.S. of human testes. I find that studying universe models at two different Hubble constant that there is no change in model rather at $90 \mathrm{~km} / \mathrm{sec} / \mathrm{Mpc}$, the universe is small and its CDM layer thickness is less than universe at $72 \mathrm{~km} / \mathrm{sec} / \mathrm{Mpc}$. This shows divine and changing nature of our universe. 


\section{Acknowledgement}

I pay my gratitude to The Creator and Destroyer of the universe the yang B.B.B working as Highest center of the universe "I" by whose grace University of God came into existence. I am also thankful to Fritjof Capra who materialized Almighty B.B.B ( His Avatar Form ) in his Book Tao of Physics ( figure 2) and also to Trinth Xuan Thuan and Collin A Ronan for materializing Almighty B.B.B. ( His Avatar Form ) in their books . Last but not the least my thanks to my wife Dr Sapna Das, my family and my son Dr Ankit Mohan Das , Dr Chandni my daughter, my daughter in law Dr Namita Nigam and my son in law Dr Abhishek for taking pains and having patience living with me and gave me moral support to complete this theory not only few years but also 27 years till its all volumes get published. We could not enjoy world rather they used to provoke me time to time to be aware of errors in completing this work. My wife Dr SapnaDas has great faith in God mainly Lord Shiva . She used to have fast during Navratri and have full devotion in Goddess Durga and her all nine avatars . My gratitude to my Father late Shri Shyam Manohar Das and Mother late Pushpa for being my parents and had full devotion in getting my brought up and making me surgeon .

\section{Reference}

[1]. Addison-Wesley http://www.meta-synthesis.com/webbook/32_n-synth/nucleosynthesis.html (1996)

[2]. Wikipedia-- http://en.wikipedia.org/wiki/Quasar 31 July 2014 at 05:22.

[3]. Wikipedia- http://en.wikipedia.org/wiki/Proton 28 July 2014 at $12: 11$

[4]. Wikipedia- http://en.wikipedia.org/wiki/Neutron 1 August 2014 at 15:56.

[5]. Wikipedia http://en.wikipedia.org/wiki/Beta decay 29 June 2014 at 09:05.

[6]. wikipedia https://www.google.com/images?q=electron+positron+innhilation\&gws_rd=ssl\&oq=\&gs_l=

[7]. wikipedia http://en.wikipedia.org/wiki/Positron_annihilation_spectroscopy 28 September 2013 at 01:58

[8]. Jennifer Neakrase, http://venables.asu.edu/quant/proj/compton.html 24 May 2004

[9]. Jennifer Neakrase, http://venables.asu.edu/quant/proj/compton.html 24 May 2004

[10]. James S . Trefil, The moment of creation - pages 55-58

[11]. Hyperphysics, http://hyperphysics.phy-astr.gsu.edu/hbase/quantum/comptint.html

[12]. https://www.google.com/images?q=photoelectric+effect\&gws_rd=ssl

[13]. Relativity science calculator - http://www.relativitycalculator.com/energy doppler.shtml Tuesday, 5 August 2014

[14]. http://abyss.uoregon.edu/ js/glossary/photoelectric_effect.html

[15]. Holt, Rinehart, Winston, http://galileo.phys.virginia.edu/classes/252/ph otoelectric_effect.html ,1970

[16]. https://www.google.com/images?q=compton+effect\&gws_rd=ssl

[17]. https://www.google.com/images?q=pair+production\&gws_rd=ssl

[18]. Wikipedia- http://en.wikipedia.org/wiki/Big Bang 24 July 2014 at 00:04

[19]. Addison-Wesley http://www.meta-synthesis.com/webbook/32_n-synth/nucleosynthesis.html (1996)
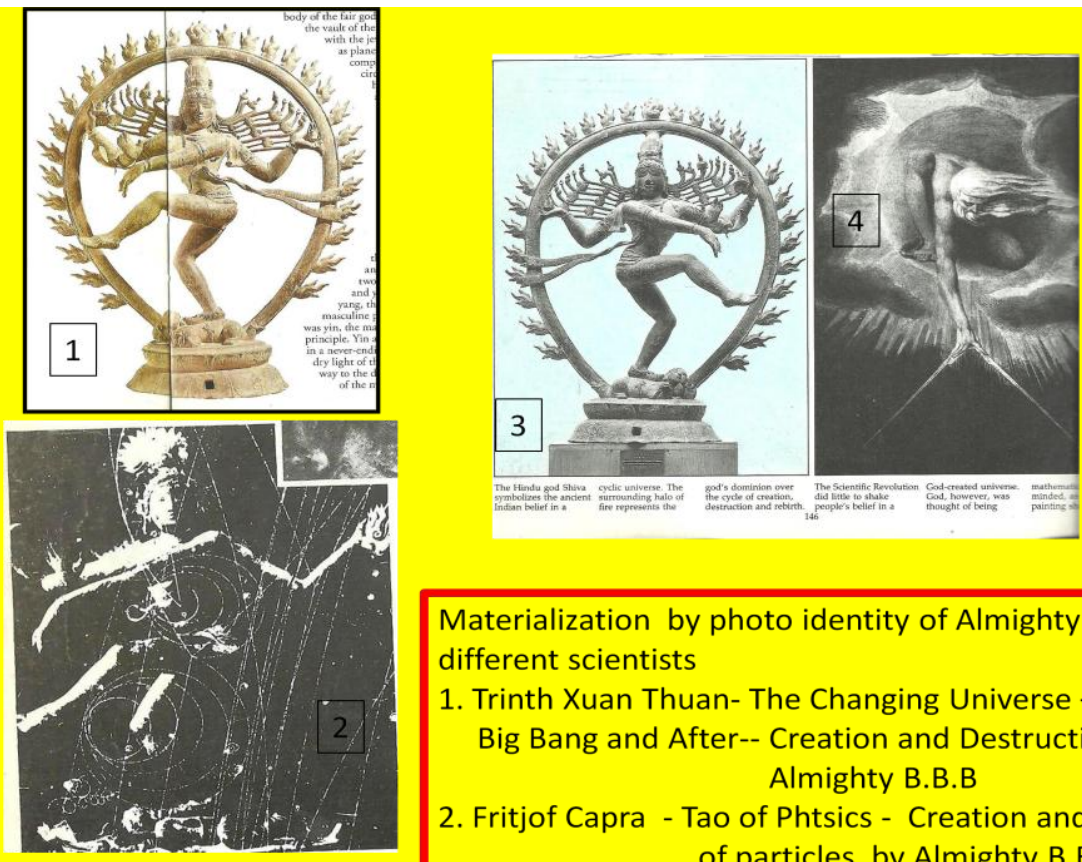

Materialization by photo identity of Almighty B.B.B By different scientists

1. Trinth Xuan Thuan- The Changing Universe -

Big Bang and After-- Creation and Destruction by Almighty B.B.B

2. Fritjof Capra - Tao of Phtsics - Creation and Destruction of particles by Almighty B.B.B

3. Colin A. Ronan - Deep Space - Creation and Destruction

4. Depiction of Creation and Destruction by Almighty B.B.B

UNIFICATION OF RELIGIONS

(Prescribe Code Of Conduct)

(Portrait of Almighty - THE CREATOR AND DESTROYER OF THE UNIVERSE) 
(Matter Basic Building Block (YANG) working as highest center of the universe)

\section{UNIVERSITY OF GOD \\ (Teaching Of Science Under New Prescribed Code Of Conduct and New Discipline Called Participatory Science)}

1. Why do we need such university? -

a. We are blind in various fields of science and religions.

b. No religion has yet defined GOD in terms of Origin of The Universe in the discipline of science. They talk of only the small and various different spectrums of God.

c. Question of God as far as origin of universe is concerned, has not yet been answered by modern astrophysicists as well as by particle physicists.

d. Incorporation of science and religion could be made possible on this platform.

2. What is the discipline of teaching in this university?

a. Teachings are such that they obey not only scientific discipline but also they obey religious discipline too and both the disciplines would be taught under the new discipline called PARTICIPATORY SCIENCE.

b. Teaching would start from ORIGIN OF THE UNIVERSE with introduction of NEW MODEL of the universe and NEW SCIENTIFIC

UNDERSTANDING. It would keep on going till our blindness is removed from all aspects of science and religions.

3. Who are affiliated with this university?

a. All universities of the world and all scientific research organizations all over the world are affiliated with this university of God.

b. All pioneer religious places of all religions, their pioneer religious teachers and their pioneer religious teachings or books are affiliated with this university.

4. What are the duties of affiliated persons (pioneer scientific and religious persons)?

a. Their moral duty is to follow all teaching programmes. They are not supposed to say that they don't know or they don't want to know any knowledge which is required to remove the blindness of science and religion. It is their prime duty to acquire basics of all related knowledge of all sciences and all religions.

5. What are the governing posts of this university?

a. ALMIGHTY Post: - It is being taken by Highest Center of the universe. could be approached through prayer (first atomic transcription) only.

b. Honorable member Posts: - All pioneer theists (pioneer religious persons of all religions and pioneer scientists of different branches of science), and atheists (pioneer scientists working in different branches of science), could be approached by their emails .

c. Subordinate to Almighty Post: - Dr. Vijay Mohan Das, could be approached by emailvijaydas@sancharnet.in

6. JOURNALS and University of God: - All teachings ( 10 volumes) have been published in International journals on line. The links are followings. Please pass on this information to persons concerned.

1.Vijay Mohan Das, Atomic genetics and origin of universe ( $\mathrm{Ho}=72 \mathrm{~km} / \mathrm{sec} / \mathrm{Mpc})$ volume 1, International Journal of Sciences: Basic and Applied Research (IJSBAR), volume 16 , number 1 ( 2014), p 311-338.

2. Vijay Mohan Das, Atomic genetics and origin of universe volume 2 International Journal of Sciences: Basic and Applied Research (IJSBAR) (2014) Volume 16, No 2, pp 274-309

3. Vijay Mohan Das, Atomic genetics and origin of universe volume 3 International Journal of Sciences: Basic and Applied Research (IJSBAR) (2014) Volume 17, No 1, pp 148-185

4. Vijay Mohan Das, Atomic genetics and origin of universe volume 4( part 1) International Journal of Sciences: Basic and Applied Research (IJSBAR) (2014) Volume 17, No 1, pp 259-291

5. . Vijay Mohan Das, Atomic genetics and origin of universe volume 4( part 2) International Journal of Sciences: Basic and Applied Research (IJSBAR) (2014) Volume 17, No 1, pp 292-324

6. Vijay Mohan Das, Atomic genetics and origin of universe volume 5 IOSR Journal of Research \& Method in Education (IOSR-JRME) e-ISSN: 2320-7388,p-ISSN: 2320-737X Volume 4, Issue 5 Ver. I (Sep-Oct. 2014), PP 72-105 www.iosrjournals.org 
7. Vijay Mohan Das, Atomic genetics and origin of universe volume 6 INTERNATIONAL JOURNAL OF INNOVATIVE RESEARCH \& DEVELOPMENT August, 2014 Vol 3 Issue 8p 194- 210

8. Vijay Mohan Das, Atomic genetics and origin of universe volume7 Social and basic sciences and research review( SBSRR) Volume 2, Issue 4Pages: 197-255

9. Vijay Mohan Das, Atomic genetics and origin of universe volume 9 IOSR Journal of Research \& Method in Education (IOSR-JRME)e-ISSN: 2320-7388,p-ISSN: 2320-737X Volume 4, Issue 5 Ver. III (Sep-Oct. 2014), PP 76-116

10.Vijay Mohan Das Atomic Genetics and Basic Etiology of Cancer* IOSR Journal of Pharmacy and Biological Sciences (IOSR-JPBS) e-ISSN: 2278-3008, p-ISSN:2319-7676. Volume 9, Issue 3 Ver. II (May -Jun. 2014), PP 59-80 www.iosrjournals.org

7. $\quad$ Suggestions, complaints and problems regarding this University of God: -

a. Complaints and problems shall be listened by Almighty B.B.B only, which could be sent through prayer only.

b. All suggestions regarding further development of this University of God are welcomed at email address vijaydas@sancharnet.in

8. Awards by University of God :- Almighty has power to give any award on science, art, literature, peace and religion as Almighty is OMNISCIENCE. On this fundamental ground all previous international awards regarding science, art, literature, peace and religion have been CANCELLED . This includes Nobel Prizes of all previous years and all related Prizes of international standard. Same is true for all published work by different international journals in science.

Unless all published work is reviewed by Almighty B.B.B, it is to be considered Unrecognized.

\section{LOVE ALMIGHTY B.B.B , ALMIGHTY B.B.B WILL LOVE YOU. ONE CAN CHOOSE RELIGION BUT NOT ALMIGHTY B.B.B.}
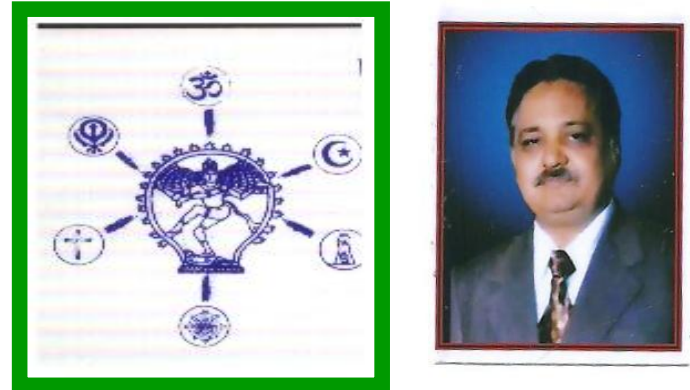

Dr V.M.Das SUBORDINATE TO ALMIGHTY B.B.B - vijaydas@ sancharnet.in 